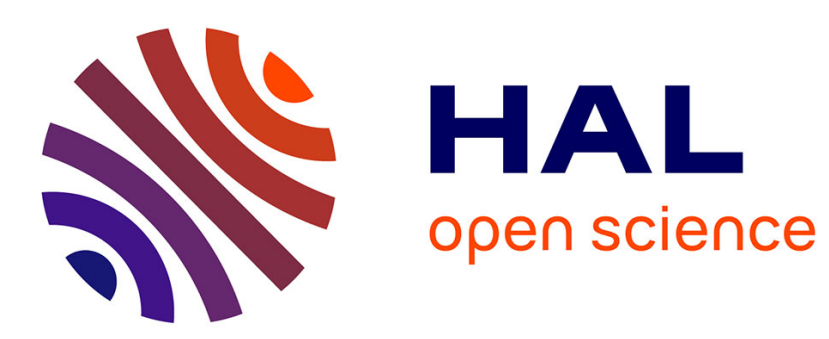

\title{
Continuum Damage-healing Mechanics with Application to Self-healing Composites
}

\author{
Ever J. Barbero, Fabrizio Greco, Paolo Lonetti
}

\section{To cite this version:}

Ever J. Barbero, Fabrizio Greco, Paolo Lonetti. Continuum Damage-healing Mechanics with Application to Self-healing Composites. International Journal of Damage Mechanics, 2005, 14 (1), pp.51-81. 10.1177/1056789505045928 . hal-00571143

\section{HAL Id: hal-00571143 \\ https://hal.science/hal-00571143}

Submitted on 1 Mar 2011

HAL is a multi-disciplinary open access archive for the deposit and dissemination of scientific research documents, whether they are published or not. The documents may come from teaching and research institutions in France or abroad, or from public or private research centers.
L'archive ouverte pluridisciplinaire HAL, est destinée au dépôt et à la diffusion de documents scientifiques de niveau recherche, publiés ou non, émanant des établissements d'enseignement et de recherche français ou étrangers, des laboratoires publics ou privés. 


\title{
Continuum Damage-healing Mechanics with Application to Self-healing Composites
}

\author{
EVER J. BARBERO* \\ Mechanical and Aerospace Engineering \\ West Virginia University, Morgantown \\ WV 26506-6106, USA \\ FABRIZIO GRECO AND PAOLO LONETTI \\ Department of Structural Engineering \\ University of Calabria, 87036 \\ Arcavacata di Rende, Cosenza, Italy
}

\begin{abstract}
The general behavior of self-healing materials is modeled including both irreversible and healing processes. A constitutive model, based on a continuum thermodynamic framework, is proposed to predict the general response of selfhealing materials. The self-healing materials' response produces a reduction in size of microcracks and voids, opposite to damage. The constitutive model, developed in the mesoscale, is based on the proposed Continuum Damage-Healing Mechanics $(\mathrm{CDHM})$ cast in a consistent thermodynamic framework that automatically satisfies the thermodynamic restrictions. The degradation and healing evolution variables are obtained introducing proper dissipation potentials, which are motivated by physically based assumptions. An efficient three-step operator slip algorithm, including healing variables, is discussed in order to accurately integrate the coupled elastoplastic-damage-healing constitutive equations. Material parameters are identified by means of simple and effective analytical procedures. Results are shown in order to demonstrate the numerical modeling of healing behavior for damaged polymer-matrix composites. Healed and not healed cases are discussed in order to show the model capability and to describe the main governing characteristics concerning the evolution of healed systems.
\end{abstract}

KEY WORDS: continuum damage-healing mechanics, self-healing composites, internal variable methods.

*Author to whom correspondence should be addressed. E-mail: ebarbero@wvu.edu

International Journal of DAMAge Mechanics, Vol. 14-January 2005 


\section{INTRODUCTION}

STructural material behavior is dominated by irreversible process $\bigcirc$ development, such as damage and residual strain release, which reduces the structural integrity and service life.

Damage and irreversible deformation phenomena affect the integrity of the material, by the creation and coalescence of microcracks, fiber breaks, fiber matrix debond, etc. The evolution of internal defects produces structural degradation, with consequent stiffness and strength reduction (Dvorak, 2000). Once microcrack development reaches the critical state, no further stress redistribution occurs, and the material rapidly reaches the failure condition (Aboudi, 1991; Pindera, 1992; Herakovich, 1998; Piggott et al., 2000).

During the last decade, modeling of dissipative phenomena have received much attention and several numerical models have been developed, which describe in various ways the inelastic response of materials. Continuum theories in a thermodynamic framework describe material degradation as stiffness and strength reduction by means of microscopic or macroscopic variables (Chow and Wang, 1987; Murakami, 1988; Chaboche, 1988; Ladeveze and Le Dantec, 1992; Voyiadjis and Deliktas, 2000). In particular, elastoplastic theories describe the slips of the material at the microscale, whereas the Continuum Damage Mechanics (CDM) provides a macroscopic representation of the microcrack and void distribution in terms of stiffness reduction. Contrary to dissipative phenomena, recent experimental observations and procedures have shown the possibility of healing several classes of materials (Miao et al., 1995; Kessler and White, 2001; Ando et al., 2002a,b; Brown et al., 2002). The healing effects can be caused by chemical, physical or biological phenomena leading to a progressive reduction of internal material defects. Experimental evidence reveals that materials can be repaired or healed in various ways and consequently the structure can be rehabilitated.

A brief literature review reveals that different healing processes have been analyzed, mainly from a phenomenological point of view, such as geological rock densification (Miao and Wang, 1994), autogenous healing of concrete or ceramic materials (Jacobsen and Sellevold, 1996; Jacobsen et al., 1996; Ramm and Biscoping, 1998; Ando, 2002a,b), microcrack regeneration in the skeleton of a biological system and so on. Numerical modeling of these processes has not been sufficiently investigated. Different models related to biological healing behavior for bone remodeling or wound skin regeneration have been developed for relatively simple cases (Adam, 1999; Simpson, 2000), but to the authors' knowledge, only a constitutive model for compaction of crushed rock salt has been proposed in a rigorous thermodynamic framework (Miao et al., 1995). 
Recently, a novel material processing technique was reported by Kessler and White (2001), White et al. (2001), and Brown et al. (2002), which describes the ability of composite materials with a polymeric-matrix to heal autonomically. Such materials are able to reduce material degradation with the aid of a healing agent by means of chemical interactions. Healing processes can be considered opposite to damage. Consequently, the system can be rehabilitated and the integrity of the material is recovered to a certain extent.

In polymer-matrix composites, the autonomic healing procedure occurs as follows. Healing agents stored in microcapsules are uniformly dispersed in the matrix material. Once a microcrack breaks a microcapsule, the healing agent is released and distributed by capillary action. The healing agent then contacts the catalyst, which is uniformly distributed in the matrix, and adhesive bonding takes place. The efficiency of the repair depends on the density of the catalyst and the microcapsules. Therefore, the microcracks evolution and the degradation processes can be controlled, and consequently, the material is self-repaired. More details about the technique and material characteristic of the healing agent can be found in the literature (Kessler and White, 2001; White et al., 2001; Brown et al., 2002; Barbero and Lonetti, 2003; Barbero et al., 2004).

The main purpose of the present paper is to generalize CDM including healing processes and consequently Continuum Damage-Healing Mechanics (CDHM) is proposed. The model is developed in a consistent thermodynamic framework and is based on the method of internal variables. The proposed constitutive model is quite general and capable of simulating different healing processes. The constitutive equations are obtained by a phenomenological thermodynamic approach using the method of internal variables. Then, an application to composite healing behavior is proposed and a numerical model is developed to predict damage and irreversible deformation processes for a self-healing fiber-reinforced lamina.

Damage and inelastic mechanisms have been discussed previously and experimentally validated (Barbero and DeVivo, 2001; Barbero and Lonetti, 2001, 2002; Lonetti et al., 2003), whereas the coupled healing-damage and irreversible deformations constitutive model is the main contribution of this paper. The proposed model predicts the distributed damage and the unrecoverable deformation in a mesoscale lamina representation, which refers to a single lamina. The orthotropic nature of composite lamina leads to a tensorial description of the healing variables, which control void and microcrack healing along different directions. An effective damaged-healed configuration is introduced, in which the body is considered without discontinuities. In order to describe the internal variable evolution, different dissipation potentials for damage, healing, and irreversible deformations (plasticity, residual strain recovery, etc.), are introduced. Coupling among 
various dissipation phenomena could be further refined as shown by $\mathrm{Abu}$ Al-Rub and Voyiadjis (2003). In the context of convex analysis, and supported by experimental observations, different limit thresholds related to damage, plasticity, and healing domains are derived. One basic assumption of the model, motivated by experimental observations, is that the healing agent acts when a sufficiently large microcrack density affects the material. In the model this occurs when the damage domain reaches the critical surface and at the same time the healing thermodynamic forces reach the healing domain. Healing is tracked by means of a thermodynamic potential that describes the evolution of healing agent.

The constitutive relationships and evolution equations define a nonlinear differential problem, which is solved by means of a proper numerical algorithm. The main equations are integrated by the Euler-backward technique, which is a stable numerical procedure to determine the actual solution by an incremental/iterative method. In particular, an elasticpredictor and Damage-healing-plasticity corrector integration scheme is used to solve the incremental nonlinear constitutive equations.

Damage and plasticity potentials are identified by means of simple but effective procedures described in Barbero and DeVivo (2001), Barbero and Lonetti (2001, 2002), and Lonetti et al. (2003). These are based on available data, which can be easily obtained by standard experimental procedures. In addition, a similar identification procedure is proposed here to identify the healing potential. Due to a lack of experimental data, the effect of allowable healing values is investigated by conducting a parametric study. A sensitivity analysis in terms of healing variables is presented in order to show the suitability of the model to predict mechanical behavior of self-healing material systems. Results are also shown in order to validate the numerical model with available experimental data for damaged polymer-matrix composites. Healed and not healed cases are discussed in order to show the capability of the model to describe the possible evolution of the self-healing composite system.

\section{THERMODYNAMIC FORMULATION}

The proposed formulation is based on generalized thermodynamics (Coleman and Gurtin, 1967; Lubliner, 1972), in which internal variables are introduced in the thermodynamic constitutive relationships to describe the inelastic processes at the current material state. The constitutive equations are thermodynamically consistent with the Clausius-Duhem inequality

$$
\boldsymbol{\sigma}: \dot{\boldsymbol{\varepsilon}}-\rho(\dot{\psi}+s \dot{\boldsymbol{T}})-\frac{\boldsymbol{q}}{T} \cdot \nabla T \geq 0
$$


where $\boldsymbol{\sigma}$ and $\boldsymbol{\varepsilon}$ are the stress and strain tensors, $\rho, \psi, s, T$, and $\boldsymbol{q}$ are mass density, specific Helmholtz free energy (HFE), specific entropy, temperature, and heat flux, respectively. A purely mechanical theory and infinitesimal deformations are assumed in the proposed model. Without loss of generality the additive strain decomposition is considered consistently with the small deformation hypotheses, by which the total deformation strain is a linear function of both elastic and plastic terms, i.e. $\varepsilon=\varepsilon^{e}+\varepsilon^{p}$. Moreover, a set of internal variables are introduced to describe both degradation and healing effects

$$
\boldsymbol{\varphi}^{d}=\boldsymbol{\varphi}^{d}(\boldsymbol{D}, \delta) \in \Im^{d}, \quad \boldsymbol{\varphi}^{p}=\boldsymbol{\varphi}^{p}\left(\boldsymbol{\varepsilon}^{p}, p\right) \in \Im^{p}, \quad \boldsymbol{\varphi}^{h}=\boldsymbol{\varphi}^{h}(\boldsymbol{H}, \mu) \in \mathfrak{\Im}^{h},
$$

where $\left(\boldsymbol{D}, \boldsymbol{\varepsilon}^{p}, \boldsymbol{H}\right)$ and $(\delta, p, \mu)$ are the tensorial and scalar variables related to damage $(d)$, plasticity $(p)$ and healing $(h)$ mechanisms and $\Im^{i}$ with $i=(d, p, h)$ are the corresponding domain spaces. It is worth noting that chemical effects related to the healing processes are only introduced from a mesoscopic point of view by means of internal variables, which describe the stiffness variation during the evolution phenomena without considering any diffusion process.

The actual thermodynamic state can be described by the HFE, $\psi: C \times$ $\Im^{d} \times \Im^{p} \times \mathfrak{\Im}^{h} \rightarrow \mathbb{R}$, which is a function of both observable and internal variables

$$
\psi=\psi\left(\varepsilon^{e}, \varphi^{d}, \varphi^{p}, \varphi^{h}\right)
$$

with $\boldsymbol{\varepsilon}^{e} \in \boldsymbol{C}$ being the admissible elastic deformation set. Substituting Equation (3) into Equation (1) and introducing the thermodynamic associated driving forces, the following constitutive equations hold

$$
\boldsymbol{\sigma}=-\rho \frac{\partial \psi}{\partial \boldsymbol{\varepsilon}^{e}}, \quad \boldsymbol{V}^{d}=-\rho \frac{\partial \psi}{\partial \boldsymbol{\varphi}^{d}}, \quad \boldsymbol{V}^{p}=-\rho \frac{\partial \psi}{\partial \boldsymbol{\varphi}^{p}}, \quad \boldsymbol{V}^{h}=\rho \frac{\partial \psi}{\partial \boldsymbol{\varphi}^{h}},
$$

with $\boldsymbol{V}^{d}=\boldsymbol{V}^{d}\left(\boldsymbol{Y}^{D}, \gamma\right), \boldsymbol{V}^{p}=\boldsymbol{V}^{p}(\tilde{\sigma}, R)$ and $\boldsymbol{V}^{H}=\boldsymbol{V}^{H}\left(\boldsymbol{Y}^{H}, \phi\right)$, where $\tilde{\sigma}$ is the stress in the effective configuration and $\boldsymbol{Y}^{D}, \boldsymbol{Y}^{H}$ are the thermodynamic forces related to damage and healing, respectively. The dissipation potential $\Xi$ is a positive defined function

$$
\Xi=\frac{\boldsymbol{V}^{d}: \dot{\boldsymbol{\varphi}}^{d}+\boldsymbol{V}^{p}: \dot{\boldsymbol{\varphi}}^{p}-\boldsymbol{V}^{h}: \dot{\boldsymbol{\varphi}}^{h}}{\text { mechanical dissipation }} \geq 0
$$

where the minus sign of the $V^{h}$ term is due to the undissipative nature of the healing process. 
The Helmholtz Free Energy is assumed to be separable as follows

$$
\psi=\psi\left(\boldsymbol{\varepsilon}^{e}, \boldsymbol{\varphi}^{d}, \boldsymbol{\varphi}^{p}, \boldsymbol{\varphi}^{h}\right)=\Phi\left(\boldsymbol{\varepsilon}^{e}, \boldsymbol{\varepsilon}^{p}, D, H\right)+\Pi(\delta, p, \mu)
$$

where $\Phi: \boldsymbol{C} \times \Im^{d} \times \Im^{h} \times \Im^{p} \rightarrow \mathbb{R}$ is the elastic deformation function, which depends on both damage and healing tensor components; whereas $\Pi: \mathbb{R} \rightarrow \mathbb{R}$ is a scalar function, which expresses the evolution of inelastic variables. The functional $\Phi$ depends on the actual value of the internal variables

$$
\Phi\left(\boldsymbol{\varepsilon}^{e}, \boldsymbol{\varepsilon}^{p}, \boldsymbol{D}, \boldsymbol{H}\right)=\frac{1}{2}\left(\boldsymbol{\varepsilon}-\boldsymbol{\varepsilon}^{p}\right): \boldsymbol{E}(\boldsymbol{D}, \boldsymbol{H}):\left(\boldsymbol{\varepsilon}-\boldsymbol{\varepsilon}^{p}\right)
$$

where $\boldsymbol{E}$ is the fourth-order damaged-healed stiffness tensor. The irreversible nature of healing and damage processes leads to a monotonically increasing evolution function. Without loss of generality, they are expressed in the following uncoupled forms

$$
\Pi(\delta, p, \mu)=\Pi^{d}(\delta)+\Pi^{p}(p)+\Pi^{h}(\mu)
$$

in which

$$
\begin{aligned}
& \Pi^{d}(\delta)=-\int_{\delta_{0}}^{\delta_{1}} \frac{\partial \psi}{\partial \delta} \cdot d \delta=\left.\left[c_{1}^{d} \delta-c_{1}^{d} c_{2}^{d} \exp \left(\delta / c_{2}^{d}\right)\right]\right|_{\delta_{0}} ^{\delta_{1}}, \quad \delta \in \Im^{\mathrm{d}},\left(c_{1}^{d}, c_{2}^{d}\right) \in \mathbb{R} \\
& \Pi^{p}(p)=-\int_{p_{0}}^{p_{1}} \frac{\partial \psi}{\partial p} \cdot d p=\left.c_{1}^{p} \frac{1}{2} p^{2}\right|_{p_{0}} ^{p_{1}}, \quad p \in \Im^{p}, c_{1}^{p} \in \mathbb{R}^{+}
\end{aligned}
$$

The damage and plasticity potentials $\Pi^{d}$ and $\Pi^{p}$ described by Barbero and Lonetti (2001, 2002), Lonetti et al. (2003), are used in order to obtain a good correspondence between the numerical and experimental data. Next, in lack of experimental data, a healing potential is assumed which is similar to the damage potential but in the corresponding thermodynamic space

$$
\Pi^{h}(\mu)=-\int_{\mu_{0}}^{\mu_{1}} \frac{\partial \psi}{\partial \mu} \cdot d \mu=\left[c_{1}^{h}\left(c_{2}^{h} \exp \left(\mu / c_{2}^{h}\right)-\mu\right)\right]_{\mu_{0}}^{\mu_{1}}, \quad \mu \in \Im^{\mathrm{H}}, c_{1}^{h}, c_{2}^{\mathrm{h}} \in \mathbb{R},
$$

This expression is motivated by intrinsic conditions of the phenomenon, in accordance with the experimental evidence, which shows that for the self-healing composite the process is basically primed by the damage 
evolution. Future developments are needed in order to completely validate Equation (10).

The material constants $\left(c_{1}^{d}, c_{2}^{d}, c_{1}^{p}, c_{1}^{h}, c_{2}^{h}\right)$ introduced in Equations (9) and (10) are identified in terms of available data as shown in "Identification of Material Parameters." The complementary laws related to the dissipation process can be expressed by homogeneous and convex potentials in terms of the associated thermodynamic forces (Hansen and Schreyer, 1994)

$$
\Upsilon^{*}\left(\boldsymbol{V}^{d}, \boldsymbol{V}^{p}, \boldsymbol{V}^{h}\right)=\Upsilon^{* d}\left(\boldsymbol{V}^{d}\right)+\Upsilon^{* p}\left(\boldsymbol{V}^{p}\right)+\Upsilon^{* h}\left(\boldsymbol{V}^{h}\right)
$$

The principle of maximum dissipation in the effective reference frame defines an equilibrium thermodynamic solution, which corresponds to a constrained optimization problem. The Lagrangian multiplier method $(L M M)$ can be used to solve the problem, where the functional

$$
\bar{\prod}=-(\Xi)+\dot{\lambda}^{p} \Upsilon_{P}^{*}\left(\boldsymbol{V}^{P}\right)+\dot{\lambda}^{d} \Upsilon_{D}^{*}\left(\boldsymbol{V}^{D}\right)+\dot{\lambda}^{h} \Upsilon_{H}^{*}\left(\boldsymbol{V}^{H}\right)
$$

depends on $\left(\dot{\lambda}^{p}, \dot{\lambda}^{d}, \dot{\lambda}^{h}\right)$ which are the Lagrangian multipliers related to plasticity, damage, and healing, respectively. In order to extremize $\bar{\prod}$, the following necessary conditions must be satisfied

$$
\frac{\partial \bar{\prod}}{\partial \boldsymbol{V}^{D}}=0, \quad \frac{\partial \bar{\prod}}{\partial \boldsymbol{V}^{P}}=0, \quad \frac{\partial \bar{\prod}}{\partial \boldsymbol{V}^{H}}=0
$$

which correspond to the plastic strain rate, damage, and healing evolution laws. The kinematic internal variables grow along the direction normal to the corresponding potential surface

$$
\dot{\varphi}^{p}=\dot{\lambda}^{P} \frac{\partial \Upsilon_{p}^{*}}{\partial \boldsymbol{V}^{P}}, \quad \dot{\varphi}^{D}=\dot{\lambda}^{D} \frac{\partial \Upsilon_{D}^{*}}{\partial \boldsymbol{V}^{D}}, \quad \dot{\varphi}^{H}=\dot{\lambda}^{H} \frac{\partial \Upsilon_{H}^{*}}{\partial \boldsymbol{V}^{H}} .
$$

\section{DAMAGE AND HEALING REPRESENTATION}

When distributed damage controls the mechanical behavior, many materials, including polymer-matrix composites, usually exhibit a quasibrittle macroscopic behavior. For example, experimental observations on polymer-matrix composite prior to failure show a continuous distribution of microcracks in the matrix. During loading, the total energy of the system is dissipated mainly into new surface formation, whereas a minor fraction is used to nucleate existing microcracks. 
The healing behavior is generated by distributed agents, which can be assumed to be uniformly distributed in the body. For example, in composite materials the microencapsulated healing agent is embedded along with a catalyst into a polymeric-matrix. The healing occurs when the microcrack path in the matrix reaches the microcapsule, and by rupture, the healing agent locally triggers a chemical polymerization reaction, leading to microcrack healing. Since the healing agent is continuously distributed, it can be analytically described by a continuous function. The same model can be applied to different materials such as crushed rock salt or bone.

Damage and healing phenomena are described at the mesoscale by internal state variables, which represent microcrack formation and healing, respectively. Two sets of tensor- and scalar-valued variables are introduced in the constitutive equations. In particular, $\boldsymbol{D}$ and $\boldsymbol{H}$ tensors describe the area change produced by microcracks and healing evolution, respectively, and scalar-valued variables $\delta$ and $\mu$ control the evolution phenomena. Lacking experimental observations to justify a more complex behavior, the evolution of the damage and healing surfaces are assumed to be isotropic.

In composite materials, microcracks and voids have preferential growth directions which coincide with the material directions. Therefore the principal directions of the damage and healing tensors are assumed to coincide with the material coordinates. In the principal reference frame, they are expressed by the following equations

$$
\boldsymbol{D}=\sum_{i=1}^{3} d_{i} \boldsymbol{n}_{i} \otimes \boldsymbol{n}_{i} \quad \boldsymbol{H}=\sum_{i=1}^{3} h_{i} \boldsymbol{n}_{i} \otimes \boldsymbol{n}_{i}
$$

where $\otimes$ represents the dyadic product; whereas $d_{i}, h_{i}$, and $\boldsymbol{n}_{\mathrm{i}}$ are the eigenvalues and the eigenvectors of the tensors $\boldsymbol{D}$ and $\boldsymbol{H}$, respectively.

Damage and healing tensors represent the net area change due to threedimensional void and microcrack distributions developed during the loading history. In the context of continuum mechanics, physical interpretation of damage and healing can be shown in Figure 1. A representative volume element of arbitrary orientation is shown in different configurations $\left(C_{0}, C_{D H}, \widetilde{C}_{F}\right)$ initial, actual (damaged-healed), and effective, respectively. Moreover, $\widetilde{C}_{F}^{*}$ and $C_{D H}^{*}$ represent the effective and damage-healed configurations free of elastic deformation, respectively. In Figure $1, \boldsymbol{F}^{e}$ represents the elastic deformation gradient. The deformation gradients $\chi_{D H}$ and $\chi_{D H}^{*}$ describe the following transformations: $\chi_{D H}: C_{D H} \rightarrow \widetilde{C}_{F}$ and $\chi_{D H}^{*}: C_{D H}^{*} \rightarrow \widetilde{C}_{F}^{*}$, where $\chi_{D H}$ and $\chi_{D H}^{*}$ have the eigenvectors coinciding with $\boldsymbol{n}_{i}$ and $\boldsymbol{n}_{i}^{*}$, respectively. The deformation of an arbitrary segment $\boldsymbol{d x _ { i }}$ to $\widetilde{\boldsymbol{d x}} \boldsymbol{x}_{i}$ between damaged-healed and effective configurations is expressed by 

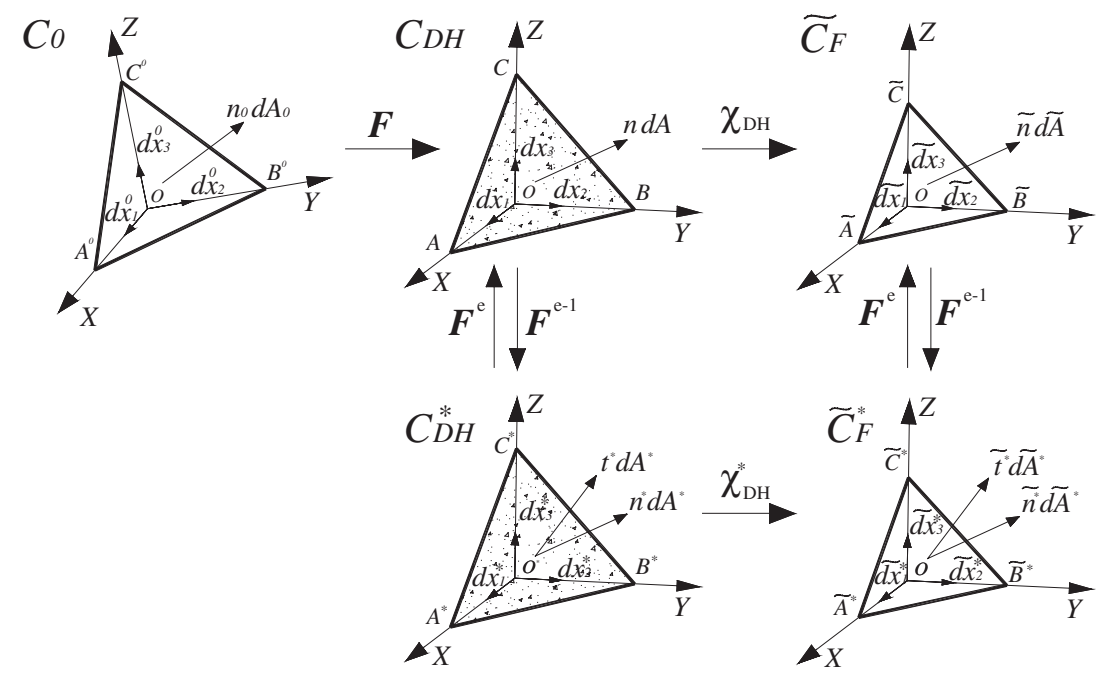

Figure 1. Clockwise from top left: undamaged, damaged-healed, effective, effective without elastic deformations, damaged-healed without elastic deformation.

introducing a transformation tensor as

$$
\widetilde{\boldsymbol{d x}} \boldsymbol{x}_{i H} \boldsymbol{d} \boldsymbol{x}_{i}, \quad \text { with } i=1,2,3
$$

From Nanson's Theorem (Ogden, 1983) specialized to the principal reference system $\left\{\boldsymbol{n}_{i}\right\}$, a generic area element is transformed by the following equations

$$
\tilde{\boldsymbol{n}} \widetilde{d S}=\frac{1}{2} \tilde{\boldsymbol{d x}} \times \tilde{\boldsymbol{d y}}=\frac{1}{2}\left(\chi_{D H} \cdot \boldsymbol{d x}\right) \times\left(\chi_{D H} \cdot \boldsymbol{d y}\right)=\operatorname{det}\left[\chi_{D H}\right]\left(\chi_{D H}^{-1 / 2}\right)^{T}\left(\chi_{D H}^{-1 / 2}\right) \boldsymbol{n} d S
$$

where $(x)$ denotes vector product. The area reduction along the principal directions can be expressed in terms of the eigenvalues of the $\boldsymbol{H}$ and $\boldsymbol{D}$ tensors as

$$
\left[\left(1-d_{i}\right)\left(1+h_{i}\right)\right] \boldsymbol{n}_{i} d S_{i}=\widetilde{\boldsymbol{n}}_{i} \widetilde{d S}_{i} \quad \text { with } i=1,2,3
$$

Here $d_{i}$ and $h_{i}$ are the eigenvalues of the damage and healing tensors along different planes and define the net area change due to degradation or healing phenomena. From an irreversible thermodynamic point of view, the evolution is based on a positive unilateral variation. From Equation (17) 
and (18), the transformation tensor $\chi_{D H}$ in the principal reference frame assumes the following expression

$$
\begin{aligned}
& \chi_{D H_{11}}=\sqrt{\frac{\left(1-d_{2}\right)\left(1+h_{2}\right)\left(1-d_{3}\right)\left(1+h_{3}\right)}{\left(1-d_{1}\right)\left(1+h_{1}\right)}} \\
& \chi_{D H_{22}}=\sqrt{\frac{\left(1-d_{1}\right)\left(1+h_{1}\right)\left(1-d_{3}\right)\left(1+h_{3}\right)}{\left(1-d_{2}\right)\left(1+h_{2}\right)}} \\
& \chi_{D H_{33}}=\sqrt{\frac{\left(1-d_{1}\right)\left(1+h_{1}\right)\left(1-d_{2}\right)\left(1+h_{2}\right)}{\left(1-d_{3}\right)\left(1+h_{3}\right)}}
\end{aligned}
$$

The effective stress $\widetilde{\boldsymbol{\sigma}}$ represents the stress associated with $\widetilde{C}_{F}$ by the same loading related to the $C_{\boldsymbol{D H}}$ configuration and corresponds to a fictitious first Piola-Kirchhoff tensor referred to $\widetilde{C}_{F}$ :

$$
\tilde{\boldsymbol{\sigma}}=\left[\operatorname{det} \chi_{D H}\right]^{-1} \chi_{D H}^{1 / 2}: \boldsymbol{\sigma}: \chi_{D H}^{1 / 2}=\boldsymbol{M}^{-1}: \boldsymbol{\sigma}
$$

where $\boldsymbol{M}$ is the effective damage tensor and corresponds to the stress tensor transformation between $\widetilde{C}_{F}$ and $C_{\boldsymbol{D} \boldsymbol{H}}$. In view of Equations (19) and (20), $\boldsymbol{M}$ is a diagonal fourth-order tensor

$$
\begin{gathered}
\boldsymbol{M}=\operatorname{diag}\left\{\Omega_{1}^{D} \Omega_{1}^{H} ; \Omega_{2}^{D} \Omega_{2}^{H} ; \Omega_{3}^{D} \Omega_{3}^{H} ; \frac{\sqrt{\Omega_{2}^{D} \Omega_{3}^{D} \Omega_{2}^{H} \Omega_{3}^{H}}}{2} ;\right. \\
\left.\frac{\sqrt{\Omega_{1}^{D} \Omega_{3}^{D} \Omega_{1}^{H} \Omega_{3}^{H}}}{2} ; \frac{\sqrt{\Omega_{1}^{D} \Omega_{2}^{D} \Omega_{1}^{H} \Omega_{2}^{H}}}{2}\right\}
\end{gathered}
$$

with

$$
\Omega_{i}^{D}=\left(1-d_{i}\right), \quad \Omega_{i}^{H}=\left(1+h_{i}\right), \quad i=1,2,3
$$

where $\tilde{\boldsymbol{\sigma}}$ is represented in contracted (Barbero, 1999), whereas the effective stress is

$$
\begin{aligned}
& \tilde{\sigma}_{11}=\frac{1}{\operatorname{det}\left(\chi_{D H}\right)} \chi_{D H_{11}} \sigma_{11}=\frac{\sigma_{11}}{\left(1-d_{1}\right)\left(1+h_{1}\right)} \\
& \widetilde{\sigma}_{22}=\frac{1}{\operatorname{det}\left(\chi_{D H}\right)} \chi_{D H_{22}} \sigma_{22}=\frac{\sigma_{22}}{\left(1-d_{2}\right)\left(1+h_{2}\right)} \\
& \tilde{\sigma}_{33}=\frac{1}{\operatorname{det}\left(\chi_{D H}\right)} \chi_{D H_{33}} \sigma_{33}=\frac{\sigma_{33}}{\left(1-d_{3}\right)\left(1+h_{3}\right)}
\end{aligned}
$$




$$
\begin{aligned}
& \tilde{\sigma}_{12}=\frac{1}{\operatorname{det}\left(\chi_{D H}\right)}\left[\chi_{D H_{11}}\right]^{1 / 2} \sigma_{12}\left[\chi_{D H_{22}}\right]^{1 / 2}=\frac{\sigma_{12}}{\sqrt{1-d_{1}} \sqrt{1-d_{2}} \sqrt{1+h_{1}} \sqrt{1+h_{2}}} \\
& \tilde{\sigma}_{13}=\frac{1}{\operatorname{det}\left(\chi_{D H}\right)}\left[\chi_{D H_{11}}\right]^{1 / 2} \sigma_{13}\left[\chi_{D H_{33}}\right]^{1 / 2}=\frac{\sigma_{13}}{\sqrt{1-d_{1}} \sqrt{1-d_{3}} \sqrt{1+h_{1}} \sqrt{1+h_{3}}} \\
& \tilde{\sigma}_{23}=\frac{1}{\operatorname{det}\left(\chi_{D H}\right)}\left[\chi_{D H_{22}}\right]^{1 / 2} \sigma_{23}\left[\chi_{D H_{33}}\right]^{1 / 2}=\frac{\sigma_{23}}{\sqrt{1-d_{2}} \sqrt{1-d_{3}} \sqrt{1+h_{2}} \sqrt{1+h_{3}}}
\end{aligned}
$$

In view of Equation (21), and according to the Principle of Equivalent Elastic Energy (Cordebois and Sidoroff, 1977), the stiffness tensor is defined by the following expression

$$
\boldsymbol{E}(\boldsymbol{D}, \boldsymbol{H})=\boldsymbol{M}: \widetilde{\boldsymbol{E}}: \boldsymbol{M}^{T}
$$

or in component form:

$$
\begin{aligned}
& E_{i j}=\left[\begin{array}{ccc}
\widetilde{E}_{11}\left(\Omega_{1}^{D} \Omega_{1}^{H}\right)^{2} & \widetilde{E}_{12} \Omega_{1}^{D} \Omega_{2}^{D} \Omega_{1}^{H} \Omega_{2}^{H} & \widetilde{E}_{13} \Omega_{1}^{D} \Omega_{3}^{D} \Omega_{1}^{H} \Omega_{3}^{H} \\
& \widetilde{E}_{22}\left(\Omega_{2}^{D} \Omega_{2}^{H}\right)^{2} & \widetilde{E}_{23} \Omega_{2}^{D} \Omega_{3}^{D} \Omega_{2}^{H} \Omega_{3}^{H} \\
\widetilde{E}_{33}\left(\Omega_{3}^{D} \Omega_{3}^{H}\right)^{2}
\end{array}\right], \quad i, j=1,3 \\
& E_{i j}=\left[\begin{array}{ccc}
\frac{\tilde{E}_{44} \Omega_{2}^{D} \Omega_{3}^{D} \Omega_{2}^{H} \Omega_{3}^{H}}{2} & 0 & 0 \\
0 & \frac{\widetilde{E}_{55} \Omega_{1}^{D} \Omega_{3}^{D} \Omega_{1}^{H} \Omega_{3}^{H}}{2} & 0 \\
0 & 0 & \frac{\widetilde{E}_{66} \Omega_{1}^{D} \Omega_{2}^{D} \Omega_{1}^{H} \Omega_{2}^{H}}{2}
\end{array}\right], i, j=4,6
\end{aligned}
$$

\section{CONSTITUTIVE EQUATIONS}

The internal variables used in the thermodynamic constitutive equations are listed in Table 1 with their associated driving forces. The Helmholtz Free Energy potential is expressed by

$$
\begin{aligned}
\psi= & \frac{1}{2}\left(\boldsymbol{\varepsilon}-\boldsymbol{\varepsilon}^{p}\right): \boldsymbol{E}:\left(\boldsymbol{\varepsilon}-\boldsymbol{\varepsilon}^{p}\right)+\left[c_{1}^{d} \delta-c_{1}^{d} c_{2}^{d} \exp \left(\delta / c_{2}^{d}\right)\right] \\
& +\frac{1}{2} c_{1}^{p} p^{2}+\left[c_{1}^{h} c_{2}^{h} \exp \left(\mu / c_{2}^{h}\right)-c_{1}^{h} \mu\right]
\end{aligned}
$$


Table 1. Observable, kinematic, and conjugate variables.

\begin{tabular}{lccc}
\hline & $\begin{array}{c}\text { Internal state variables } \\
\text { Ouantity }\end{array}$ & $\begin{array}{c}\text { Kinematic } \\
\text { variables }\end{array}$ & $\begin{array}{c}\text { Thermodynamic } \\
\text { forces }\end{array}$ \\
\hline Strain & $\varepsilon$ & & \\
Temperature & $T$ & $D$ & $Y^{D}$ \\
Damage & & $\delta$ & $\gamma$ \\
Damage evolution & $\varepsilon^{p}$ & $\sigma$ \\
Plastic strain & $p$ & $R$ \\
Hardening & $H$ & $Y^{H}$ \\
Healing & $\mu$ & $\phi$ \\
Healing evolution & & & \\
\hline
\end{tabular}

whereas the thermodynamic forces are defined by Equation (4)

$$
\begin{gathered}
\boldsymbol{\sigma}=-\rho \frac{\partial \psi}{\partial \boldsymbol{\varepsilon}^{e}}=\boldsymbol{E}:\left(\boldsymbol{\varepsilon}-\boldsymbol{\varepsilon}^{p}\right) \\
\tilde{\boldsymbol{\sigma}}=\boldsymbol{M}^{-1}: \boldsymbol{\sigma} \\
\boldsymbol{Y}^{D}=-\rho \frac{\partial \psi}{\partial \boldsymbol{D}}=-\frac{1}{2}\left(\boldsymbol{\varepsilon}-\boldsymbol{\varepsilon}^{p}\right):\left(\frac{\partial \boldsymbol{E}}{\partial \boldsymbol{D}}\right):\left(\boldsymbol{\varepsilon}-\boldsymbol{\varepsilon}^{p}\right) \\
\boldsymbol{Y}^{H}=\rho \frac{\partial \psi}{\partial \boldsymbol{H}}=\frac{1}{2}\left(\boldsymbol{\varepsilon}-\boldsymbol{\varepsilon}^{p}\right):\left(\frac{\partial \boldsymbol{E}}{\partial \boldsymbol{H}}\right):\left(\boldsymbol{\varepsilon}-\boldsymbol{\varepsilon}^{p}\right) \\
\boldsymbol{R}=-\rho \frac{\partial \psi}{\partial p}=-c_{1}^{p} p \\
\gamma=-\rho \frac{\partial \psi}{\partial \delta}=c_{1}^{d} \cdot\left[\exp \left(\delta / c_{2}^{d}\right)-1\right] \\
\phi=\rho \frac{\partial \psi}{\partial \mu}=c_{1}^{h} \cdot\left[\exp \left(\mu / c_{2}^{h}\right)-1\right]
\end{gathered}
$$

The healing thermodynamic forces concept can be illustrated for a simple tensile stress cycle by considering the balance of dissipated energy. A generic stress-strain curve is shown in Figure 2, in which damage and healing are assumed to increase with increasing stress. The total energy dissipated during a cycle is the area $O \widehat{A B C} E$. The recovery energy due to healing effects and the dissipation energy due to damage and plasticity are defined by areas $\widehat{C D E}, \widehat{B D E}$, and $\widehat{O A F E}$, respectively. The healing phenomena is generated by internal energy production, which is obtained by spending chemical energy stored in the healing agent or provided externally by a 


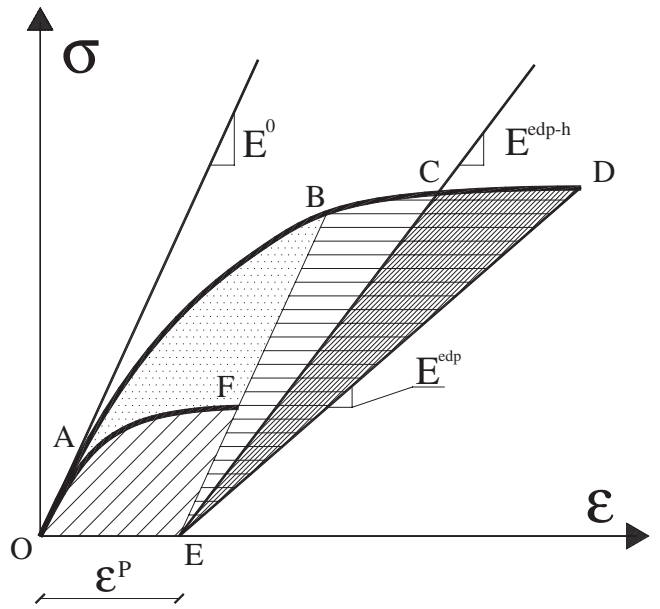

Figure 2. Stress-strain curve: Elasto-plastic-damage and elasto-plastic-damage-healing.

sinterization process or biological bone repair. The healing process increases the stiffness of the material. In view of Equation (5), for a uniaxial stress state, damage and healing energies are dissipated during $\overline{\mathrm{BCD}}$ and $\overline{\mathrm{CD}}$ paths, respectively and they are mathematically written as

$$
\begin{aligned}
& \Xi^{D}=\left.\int_{0}^{d} Y^{D}\right|_{h=0} d D=C_{11} \sigma^{2}\left[-\frac{1}{2}+\frac{1}{2(1-d)^{2}}\right] \geq 0 \\
& \Xi^{H}=\left.\int_{0}^{h} Y^{H}\right|_{d=\mathrm{const}} d H=\frac{C_{11} \sigma^{2}}{(1-d)^{2}}\left[\frac{1}{2}-\frac{1}{2(1-h)^{2}}\right] \leq 0
\end{aligned}
$$

Equations (34) represent areas $B \widehat{B D} E$ and $\widehat{D C E}$ in which uncoupled damage and healing growth are assumed. Damage energy production $\Xi^{D}$ describes material degradation and it is a strictly positive definite function. The healing phenomena generate internal energy production, which is opposite to the damage dissipation.

In view of Equations (27)-(33) under the hypothesis of decoupling between different processes and according to the Clausius-Duhem inequality, the thermodynamic dissipation function has to be necessarily positive

$$
\begin{aligned}
& \Xi^{P}=\tilde{\sigma}: \tilde{\dot{\varepsilon}}^{p}+\boldsymbol{R} \cdot \dot{\boldsymbol{p}} \geq 0 \\
& \Xi^{D}=\boldsymbol{Y}^{d}: \dot{\boldsymbol{D}}+\gamma \cdot \dot{\delta} \geq 0 \\
& \Xi^{H}=-\boldsymbol{Y}^{h}: \dot{\boldsymbol{H}}-\phi \cdot \dot{\mu} \leq 0
\end{aligned}
$$


where $\Xi^{P}, \Xi^{D}$, and $\Xi^{H}$ are the dissipation functions related to plasticity, damage, and healing processes, respectively, with $\Xi=\Xi^{P}+\Xi^{D}+\Xi^{H}$ being the total dissipation function. The total dissipation is always positive because healing phenomena are activated only when the magnitude of the microcrack distribution is significant (White et al., 2001) and the efficiency of the healing mechanism is less than $100 \%$. A $100 \%$ healing efficiency would correspond to perfect healing, where all damage dissipation would be recovered (i.e. $\Xi^{D}=\Xi^{H}$ ).

According to the method of local state, the evolution laws can be derived from dissipation potentials, whose existence is postulated a priori. Damage and plasticity potentials previously proposed by Barbero and DeVivo (2001), Barbero and Lonetti (2001, 2002), Lonetti et al. (2003), have shown good correspondence between experimental data and numerical results. These are

$$
\begin{aligned}
f^{d}= & \left(\boldsymbol{Y}^{D}: \boldsymbol{J}^{D}: \boldsymbol{Y}^{D}\right)^{1 / 2}-\gamma(\delta)-\gamma_{0} \\
f^{p}(\widetilde{\boldsymbol{\sigma}})= & g^{p}(\widetilde{\boldsymbol{\sigma}})=f_{1} \widetilde{\sigma}_{1}+f_{2} \widetilde{\sigma}_{2}+f_{11} \widetilde{\sigma}_{1}^{2}+f_{22} \widetilde{\sigma}_{2}^{2}+2 f_{12} \widetilde{\sigma}_{1} \widetilde{\sigma}_{2} \\
& +f_{44} \widetilde{\sigma}_{4}^{2}+f_{55} \widetilde{\sigma}_{5}^{2}+f_{66} \widetilde{\sigma}_{6}^{2}-R(p)-R_{0}
\end{aligned}
$$

where $\gamma_{0}$ and $R_{0}$ are the plasticity and damage thresholds, $\boldsymbol{J}^{D}$ is a fourthorder damage characteristic tensor and $f_{i}$ are material parameters. For healing, an evolution potential similar to the damage one is proposed

$$
f^{H}=\left(\boldsymbol{Y}^{H}: \boldsymbol{J}^{H}: \boldsymbol{Y}^{H}\right)^{1 / 2}-\phi(\mu)-\phi_{0}
$$

where $\phi_{0}$ is the healing threshold and $\boldsymbol{J}^{H}$ is a fourth-order healing tensor. The previous assumptions will be clarified "Identification of Material Parameters." From Equation (14), the evolution vectors are assumed to develop along the normal direction of the corresponding potential surface

$$
\begin{aligned}
& \dot{\boldsymbol{\varphi}}^{d}=\left[\begin{array}{c}
\dot{\delta} \\
\dot{\boldsymbol{D}}
\end{array}\right]=\dot{\lambda}^{d} \nabla_{, V^{d}} f^{d}=\dot{\lambda}^{d}\left[\begin{array}{c}
-1 \\
\frac{\boldsymbol{J}^{D}: \boldsymbol{Y}^{D}}{\sqrt{\boldsymbol{Y}^{D} \cdot\left(\boldsymbol{J}^{D}: \boldsymbol{Y}^{D}\right)}}
\end{array}\right] \\
& =\dot{\lambda}^{d}\left[\frac{1}{\Delta^{d}}\left[\begin{array}{ccc}
J_{11}^{D} Y_{1}^{D} & 0 & 0 \\
0 & J_{22}^{D} Y_{2}^{D} & 0 \\
0 & 0 & J_{33}^{D} Y_{3}^{D}
\end{array}\right]\right] \\
& \dot{\boldsymbol{\varphi}}^{p}=\left[\begin{array}{c}
\dot{p} \\
\tilde{\dot{\varepsilon}}^{p}
\end{array}\right]=\dot{\lambda}^{p} \nabla_{V^{p}} g^{p}
\end{aligned}
$$




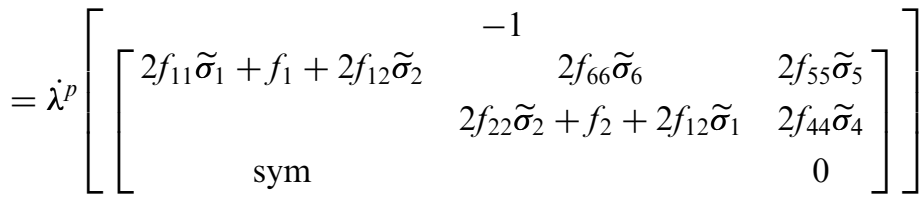

$$
\begin{aligned}
& \dot{\boldsymbol{\varphi}}^{H}=\left[\begin{array}{c}
\dot{\mu} \\
\dot{\boldsymbol{H}}
\end{array}\right]=\dot{\lambda}^{H} \nabla_{, V^{H}} f^{H}=\dot{\lambda}^{H}\left[\frac{\boldsymbol{J}^{H}: \boldsymbol{Y}^{H}}{\sqrt{\boldsymbol{Y}^{H} \cdot\left(\boldsymbol{J}^{H}: \boldsymbol{Y}^{H}\right)}}\right] \\
& =\dot{\lambda}^{H}\left[\frac{1}{\Delta^{H}}\left[\begin{array}{ccc}
J_{11}^{H} Y_{1}^{H} & 0 & 0 \\
0 & J_{22}^{H} Y_{2}^{H} & 0 \\
0 & 0 & J_{33}^{H} Y_{3}^{H}
\end{array}\right]\right] \\
& \text { with } \Delta^{D, H}=\sqrt{J_{11}^{D, H}\left(Y_{1}^{D, H}\right)^{2}+J_{22}^{D, H}\left(Y_{2}^{D, H}\right)^{2}+J_{33}^{D, H}\left(Y_{3}^{D, H}\right)^{2}}
\end{aligned}
$$

\section{Inelastic and Healing Domain}

The main expressions from previous plasticity and damage formulations are summarized here (Barbero and DeVivo, 2001; Barbero and Lonetti, 2001, 2002; Lonetti et al., 2003). Damage and plasticity were based on experimental observations of acoustic emissions, which indicate marked damage and plasticity thresholds (Liu et al., 1997; Gong et al., 2000). The initial threshold values are represented by $\gamma_{0}$ and $R_{0}$ for damage and plasticity, respectively. An anisotropic damage criterion for polymeric composite materials is written in terms of tensorial parameters

$$
g^{d}=\left(\boldsymbol{Y}^{\boldsymbol{D}}: \boldsymbol{J}^{\boldsymbol{D}}: \boldsymbol{Y}^{\boldsymbol{D}}\right)^{1 / 2}+\left(\left|\boldsymbol{H}^{\boldsymbol{D}} \cdot \boldsymbol{Y}^{\boldsymbol{D}}\right|\right)^{1 / 2}-\gamma(\delta)-\gamma_{0}
$$

where, $g^{d}: \Im^{\prime d} \rightarrow \mathbb{R}^{+}$. Substituting Equations (29) and (27) in Equation (39), the damage domain in the stress space has the same shape of the Tsai$\mathrm{Wu}$ surface, which is a widely accepted failure surface (Barbero, 1999). The procedure for identification of the $J^{D}$ and $H^{D}$ tensors is based on comparison of Equations (39) and (32) with the Tsai-Wu surface. The plasticity domain is identical to the plasticity potential (second of Equation (36)), but written in the effective Damage-Healing configuration in order to recover the coupling between different modes (damage, healing, plasticity). In Equation (36), $R(p)$ is the isotropic evolution function and $f_{i}$ are material parameters that depend on experimental values obtained from testing a 
single composite lamina. Analogous to damage processes, a healing domain is introduced that is similar in expression to the damage one, but written in different thermodynamic force space

$$
g^{H}=\left(\boldsymbol{Y}^{H}: \boldsymbol{J}^{H}: \boldsymbol{Y}^{H}\right)^{1 / 2}+\left(\left|\boldsymbol{H}^{H} \cdot \boldsymbol{Y}^{H}\right|\right)^{1 / 2}-\phi(\mu)-\phi_{0}
$$

where $\boldsymbol{J}^{H}$ and $\boldsymbol{H}^{H}$ are tensor-valued variables that define the healing shape surface and $\phi(\mu)$ is the healing evolution function (Equation (33)). The healing surface is motivated by experimental evidence. In particular, healing phenomena start when significant microcrack distribution is observed. Subsequently, the material is rehabilitated with a finite efficiency depending on microcapsule and catalyst density. Therefore, $\phi_{0}$ controls the beginning of healing, and the healing surface defines the limit space related to possible healing production. Moreover, the analogy with the $g^{d}$ surface is motivated by experimental observations that show how healing phenomena depend on microcrack and void distribution. Therefore, the proposed model predicts healing evolution by introducing a healing surface, which is obviously similar to the damage surface. Healing processes start at those points at which a considerable damage value is observed. In the model this occurs when the healing thermodynamic forces reach the corresponding healing surface.

\section{Evolution Equations}

The kinematic evolution laws are derived using the principle of maximum dissipation in a consistent and generalized thermodynamic approach. The solution is obtained by solving a nonlinear system equation using an incremental, iterative process. For a generic thermodynamic state, the Kuhn-Tucker Optimality (KTO) conditions must be satisfied

$g^{D}\left(\boldsymbol{V}^{D}\right)=d g^{D}\left(\boldsymbol{V}^{D}\right)=0, \quad g^{P}\left(\boldsymbol{V}^{P}\right)=d g^{P}\left(\boldsymbol{V}^{P}\right)=0, \quad g^{H}\left(\boldsymbol{V}^{H}\right)=d g^{H}\left(\boldsymbol{V}^{H}\right)=0$

Substituting Equations (27)-(33) and (38) in (41), a system of linear equations in the unknown quantities $\dot{\lambda}^{d}, \dot{\lambda}^{p}$, and $\dot{\lambda}^{h}$ is obtained

$$
\begin{aligned}
{[\boldsymbol{A}][\bar{\lambda}]+[\boldsymbol{b}] } & =\mathbf{0} \\
\boldsymbol{A} & =\left[\begin{array}{lll}
a_{11} & a_{12} & a_{13} \\
a_{21} & a_{22} & a_{23} \\
a_{31} & a_{32} & a_{33}
\end{array}\right], \quad \overline{\boldsymbol{\lambda}}=\left[\begin{array}{c}
\dot{\lambda}^{d} \\
\dot{\lambda}^{p} \\
\dot{\lambda}^{h}
\end{array}\right], \quad \boldsymbol{b}=\left[\begin{array}{c}
b_{11} \\
b_{22} \\
b_{33}
\end{array}\right]
\end{aligned}
$$


with

$$
\begin{aligned}
& a_{11}=\left(\frac{\partial g^{d}}{\partial \boldsymbol{Y}^{D}} \frac{\partial \boldsymbol{Y}^{D}}{\partial \boldsymbol{D}} \nabla_{, Y^{D}} f^{d}+\frac{\partial g^{d}}{\partial \gamma} \frac{\partial \gamma}{\partial \delta} \nabla_{, \gamma} f^{d}\right), \\
& a_{12}=\left(\frac{\partial g^{d}}{\partial \boldsymbol{Y}^{D}} \frac{\partial \boldsymbol{Y}^{D}}{\partial \boldsymbol{\varepsilon}^{p}} \boldsymbol{M}^{-1} \nabla_{, \tilde{\sigma}} g^{p}\right), \\
& a_{13}=\left(\frac{\partial g^{d}}{\partial \boldsymbol{Y}^{D}} \frac{\partial \boldsymbol{Y}^{D}}{\partial \boldsymbol{H}} \nabla_{, Y^{H}} f^{h}\right) \\
& b_{11}=\left(\frac{\partial g^{d}}{\partial \boldsymbol{Y}^{D}} \frac{\partial \boldsymbol{Y}^{D}}{\partial \boldsymbol{\varepsilon}} d \boldsymbol{\varepsilon}\right), \\
& a_{21}=\frac{\partial g^{p}}{\partial \widetilde{\boldsymbol{\sigma}}} \frac{\partial}{\partial \boldsymbol{D}}\left[\boldsymbol{M}^{-1} \boldsymbol{\sigma}\right] \nabla_{, Y^{D} f^{d},} \\
& a_{22}=\left(\frac{\partial g^{p}}{\partial \widetilde{\boldsymbol{\sigma}}} \boldsymbol{M}^{-1} \frac{\partial \boldsymbol{\sigma}}{\partial \varepsilon^{p}} \boldsymbol{M}^{-1} \nabla_{, \sigma^{g}}{ }^{p}+\frac{\partial g^{p}}{\partial R} \frac{\partial R}{\partial p} \nabla_{, R} g^{p}\right), \\
& a_{23}=\frac{\partial g^{p}}{\partial \widetilde{\boldsymbol{\sigma}}} \frac{\partial}{\partial \boldsymbol{H}}\left[\boldsymbol{M}^{-1} \boldsymbol{\sigma}\right] \nabla_{, Y^{H}} f^{h}, \\
& b_{22}=\frac{\partial g^{p}}{\partial \widetilde{\boldsymbol{\sigma}}} \boldsymbol{M}^{-1} \frac{\partial \boldsymbol{\sigma}}{\partial \boldsymbol{\varepsilon}} d \boldsymbol{\varepsilon}, \\
& a_{33}=\left(\frac{\partial g^{h}}{\partial \boldsymbol{Y}^{H}} \frac{\partial \boldsymbol{Y}^{H}}{\partial \boldsymbol{H}} \nabla_{, Y^{H}} f^{h}+\frac{\partial g^{h}}{\partial \phi} \frac{\partial \phi}{\partial \mu} \nabla_{, \phi} f^{h}\right), \\
& a_{32}=\left(\frac{\partial g^{h}}{\partial \boldsymbol{Y}^{H}} \frac{\partial \boldsymbol{Y}^{H}}{\partial \boldsymbol{\varepsilon}^{p}} \boldsymbol{M}^{-1} \nabla_{, \sigma^{p}}\right), \\
& a_{31}=\left(\frac{\partial g^{h}}{\partial \boldsymbol{Y}^{H}} \frac{\partial \boldsymbol{Y}^{H}}{\partial \boldsymbol{D}} \nabla_{, Y^{D}} f^{d}\right), \\
& b_{33}=\left(\frac{\partial g^{h}}{\partial \boldsymbol{Y}^{H}} \frac{\partial \boldsymbol{Y}^{H}}{\partial \boldsymbol{\varepsilon}} d \boldsymbol{\varepsilon}\right) .
\end{aligned}
$$

Moreover, substituting Equations (43) into Equation (42), it is possible to derive the incremental relationships

$$
\begin{aligned}
{\left[\begin{array}{c}
\boldsymbol{d} \boldsymbol{D} \\
\boldsymbol{d} \boldsymbol{\varepsilon}^{p} \\
\boldsymbol{d} \boldsymbol{H}
\end{array}\right] } & =\operatorname{diag}\left[\begin{array}{c}
\nabla_{, Y^{D}} f^{d} \\
\nabla_{, \widetilde{\sigma}^{p}} \\
\nabla_{Y^{H}} f^{d}
\end{array}\right]\left[\begin{array}{c}
d \lambda^{d} \\
d \lambda^{p} \\
d \lambda^{h}
\end{array}\right] \\
& =\operatorname{diag}\left[\begin{array}{c}
\nabla_{, Y^{D}} f^{d} \\
\nabla_{, \widetilde{\sigma}^{p}} \\
\nabla_{Y^{H}} f^{h}
\end{array}\right][A]^{-1}\left[\begin{array}{c}
\frac{\partial g^{d}}{\partial \boldsymbol{Y}^{D}} \frac{\partial \boldsymbol{Y}^{D}}{\partial \boldsymbol{\varepsilon}} \\
\frac{\partial g^{p}}{\partial \widetilde{\boldsymbol{\sigma}}} \boldsymbol{M}^{-1} \frac{\partial \boldsymbol{\sigma}}{\partial \boldsymbol{\varepsilon}} \\
\frac{\partial g^{h}}{\partial \boldsymbol{Y}^{H}} \frac{\partial \boldsymbol{Y}^{H}}{\partial \boldsymbol{\varepsilon}}
\end{array}\right] d \varepsilon=\left[\begin{array}{c}
\aleph^{d} \\
\aleph^{p} \\
\aleph^{h}
\end{array}\right][d \varepsilon]
\end{aligned}
$$


where $\aleph^{i}$, with $j=d, p, h$, represent the stiffness tensor contributions related to damage, plasticity, and healing mechanisms. Using Equations (24), (27) and (28), the incremental stress in the effective configuration can be expressed in the following form

$$
d \widetilde{\boldsymbol{\sigma}}=d \boldsymbol{M}^{-1}: \boldsymbol{\sigma}+\boldsymbol{M}^{-1}: d \boldsymbol{\sigma}=\left[\frac{d \boldsymbol{M}}{d \boldsymbol{D}}: d \boldsymbol{D}+\frac{d \boldsymbol{M}}{d \boldsymbol{H}}: d \boldsymbol{H}\right]^{-1}: \boldsymbol{\sigma}+\boldsymbol{M}^{-1}: d \boldsymbol{\sigma},
$$

where

$$
\widetilde{\boldsymbol{\sigma}}=\widetilde{\boldsymbol{E}}:\left(\widetilde{\boldsymbol{\varepsilon}}-\widetilde{\boldsymbol{\varepsilon}}^{p}\right), \quad d \boldsymbol{M}=\frac{d \boldsymbol{M}}{d \boldsymbol{D}}: d \boldsymbol{D}+\frac{d \boldsymbol{M}}{d \boldsymbol{H}}: d \boldsymbol{H} .
$$

Substituting Equations (46) and (24) in Equation (45), the incremental stress-strain relationship in the actual configuration is written as

$$
d \boldsymbol{\sigma}=\boldsymbol{E}_{T}^{\mathrm{edph}}: d \boldsymbol{\varepsilon}
$$

with

$$
\begin{aligned}
\boldsymbol{E}_{T}^{e p d h}= & \boldsymbol{E}_{T}^{e p}+\boldsymbol{E}_{T}^{d h} \\
\boldsymbol{E}_{T}^{e p}= & \boldsymbol{M}: \boldsymbol{E}: \boldsymbol{M}\left(\boldsymbol{I}-\aleph^{p}\right)_{\text {elasto-plasticity }} \\
\boldsymbol{E}_{T}^{d h}= & \left(\boldsymbol{M}: \boldsymbol{E}:\left(\frac{d \boldsymbol{M}}{d \boldsymbol{D}}: \aleph^{d}+\frac{d \boldsymbol{M}}{d \boldsymbol{H}}: \aleph^{h}\right)\right. \\
& \left.\quad \boldsymbol{M}:\left(\frac{d \boldsymbol{M}^{-1}}{d \boldsymbol{D}}: \aleph^{d}: \boldsymbol{D}+\frac{d \boldsymbol{M}^{-1}}{d \boldsymbol{H}}: \aleph^{h}: \boldsymbol{H}\right)\right)_{\text {damage-healing }}
\end{aligned}
$$

where $\boldsymbol{E}_{T}^{e p}$ and $\boldsymbol{E}_{T}^{d h}$ represent the elastoplastic and healing-damage contributions.

\section{INTEGRATION PROCEDURE}

The solution is obtained by an incremental-iterative procedure based on a return-mapping algorithm (Ju, 1989; Crisfield, 1991; Luccioni et al., 1996). In particular, a predictor-corrector scheme is used. The initial deformation increment is considered perfectly elastic or elastic-damaged, so that the stress variation is a function of the initial elastic-damaged stiffness tensor, as $\boldsymbol{\sigma}^{i}=\boldsymbol{\sigma}^{i-1}+E_{T}^{e} \Delta \boldsymbol{\varepsilon}$. The total deformation increment is divided into an elastic 
and a plastic term. Subsequently, the stiffness matrix is transformed by degradation and/or healing variables. The solution at step $n+1$ is subjected to the following evolution restrictions for damage, plasticity, and healing effects

$$
\begin{array}{lll}
\dot{\lambda}^{d} \geq 0 & \dot{\lambda}^{d} \cdot g^{d}\left(\boldsymbol{Y}_{n+1}^{D}, \gamma_{n+1}\right) \leq 0 & g^{d}\left(\boldsymbol{Y}_{n+1}^{D}, \gamma_{n+1}\right) \leq 0 \\
\dot{\lambda}^{p} \geq 0 & \dot{\lambda}^{p} \cdot g^{p}\left(\widetilde{\boldsymbol{\sigma}}_{n+1}, R_{n+1}\right) \leq 0 & g^{p}\left(\widetilde{\boldsymbol{\sigma}}_{n+1}, R_{n+1}\right) \leq 0 \\
\dot{\lambda}^{h} \geq 0 & \dot{\lambda}^{h} \cdot g^{h}\left(\boldsymbol{Y}_{n+1}^{H}, \phi_{n+1}\right) \leq 0 & g^{h}\left(\boldsymbol{Y}_{n+1}^{H}, \phi_{n+1}\right) \leq 0
\end{array}
$$

which correspond to Kuhn-Tucker Optimality conditions. The initial Lagrangian Multipliers solution obtained by Equation (42) corresponds to a thermodynamic state that does not necessarily satisfy Equation (49). Therefore, an iterative procedure is needed to solve the nonlinear problem. Using the constitutive equations, the surface domain at the $i+1$ th iteration can be expressed to the first order by Taylor expansion and the nonlinear system is reduced to the following linearized equations

$$
\nabla\left[\begin{array}{l}
g^{d} \\
g^{d} \\
g^{h}
\end{array}\right]\left[\begin{array}{c}
\Delta \lambda^{d} \\
\Delta \lambda^{p} \\
\Delta \lambda^{h}
\end{array}\right]+\left[\begin{array}{c}
g^{d} \\
g^{p} \\
g^{h}
\end{array}\right]_{i+1}=0
$$

in which $\Delta \lambda^{i}$ are the unknown quantities (see Appendix I). Between the $n$ and $n+1$ steps, the kinematic and thermodynamic forces are updated by the following incremental relationships

$$
\begin{aligned}
\boldsymbol{\sigma}_{n+1}= & \boldsymbol{\sigma}_{n+1}^{k}+\Delta \boldsymbol{\sigma}_{n}^{k+1} \\
\Delta \boldsymbol{\sigma}_{n}^{k+1}= & {\left[\widetilde{\boldsymbol{E}}\left(-\boldsymbol{M}^{-1} \Delta \lambda^{p} \frac{\partial g^{p}}{\partial \widetilde{\boldsymbol{\sigma}}}\right)\right.} \\
& \left.+\left(\frac{\partial\left[\boldsymbol{M}^{-1} \widetilde{\boldsymbol{E}}\right]}{\partial \boldsymbol{D}} \Delta \lambda^{d} \frac{\partial f^{d}}{\partial \boldsymbol{D}}+\frac{\partial\left[\boldsymbol{M}^{-1} \widetilde{\boldsymbol{E}}\right]}{\partial \boldsymbol{H}} \Delta \lambda^{h} \frac{\partial f^{h}}{\partial \boldsymbol{H}}\right)\left(\boldsymbol{\varepsilon}-\boldsymbol{\varepsilon}^{p}\right)\right]\left.\right|_{n} ^{k+1} \\
\boldsymbol{D}_{n+1}= & \boldsymbol{D}_{n+1}^{k}+\Delta \boldsymbol{D}_{n}^{k+1}=\boldsymbol{D}_{n}+\left.\Delta \lambda^{d} \frac{\partial f^{d}}{\partial \boldsymbol{D}}\right|_{n} ^{k+1} \\
\widetilde{\boldsymbol{\sigma}}_{n+1}= & \boldsymbol{M}^{-1}\left(\boldsymbol{D}_{n+1}\right) \boldsymbol{\sigma}_{n+1} \\
\widetilde{\boldsymbol{\varepsilon}}_{n+1}^{p}= & \widetilde{\boldsymbol{\varepsilon}}_{n+1}^{p(k)}+\Delta \widetilde{\boldsymbol{\varepsilon}}_{n+1}^{p(k+1)}=\widetilde{\boldsymbol{\varepsilon}}_{n+1}^{p(k)}+\left.\Delta \lambda^{p} \frac{\partial g^{p}}{\partial \widetilde{\boldsymbol{\sigma}}}\right|_{n} ^{k+1}
\end{aligned}
$$




$$
\begin{aligned}
\boldsymbol{H}_{n+1} & =\boldsymbol{H}_{n+1}^{k}+\Delta \boldsymbol{H}_{n}^{k+1}=\boldsymbol{H}_{n}+\left.\Delta \lambda^{h} \frac{\partial f^{h}}{\partial \boldsymbol{H}}\right|_{n} ^{k+1} \\
\mu_{n+1} & =\mu_{n+1}^{k}+\Delta \mu_{n+1}^{k+1}=\mu_{n+1}^{k}+\Delta \lambda^{h} \\
\delta_{n+1} & =\delta_{n+1}^{k}+\Delta \delta_{n+1}^{k+1}=\delta_{n+1}^{k}+\Delta \lambda^{d} \\
p_{n+1} & =p_{n+1}^{k}+\Delta p_{n+1}^{k+1}=p_{n+1}^{p}+\Delta \lambda^{p}
\end{aligned}
$$

\section{IDENTIFICATION OF MATERIAL PARAMETERS}

The material parameters in the constitutive equations are determined in terms of experimentally observed material behavior. The identification is done by solving a nonlinear system of equations obtained by comparing the healing domain (Equation (40)) and a classical Tsai-Wu surface (Barbero, 1999) in stress space. The identification of the healing parameters is shown here, whereas damage and plasticity parameter identification is described by Lonetti et al. (2003), Barbero and Lonetti (2001, 2002).

As shown by experimental observations, healing starts only when a significant microcrack distribution occurs in the matrix. Moreover, it is well known that healing processes are generated by microcrack evolution. Therefore, the basic idea is to assume a healing surface similar in expression to the damaged one. The healing potentials described by Equations (40) and (37) involve two characteristic tensors $\boldsymbol{J}^{H}$ and $\boldsymbol{H}^{H}$ that define the domain shape and the evolution of kinematic variables (Equation (33)). The healing scalar function $\phi$ represents isotropic growth of the healing domain, where the scalar $\phi_{0}$ corresponds to the initial healing threshold. Damage and healing surfaces in the corresponding thermodynamic force spaces are shown in Figure 3 , with healing and damage hardening given by $\left(\phi_{0}+\phi, \gamma_{0}+\gamma\right)$.

Identification of the characteristic healing tensors is provided by the following nonlinear system of equations. Considering a uniaxial ultimate

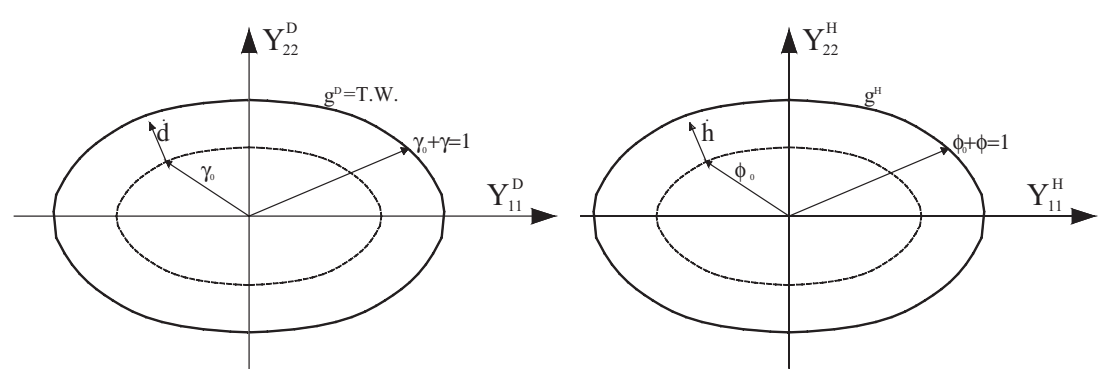

Figure 3. Schematic healing-damage domain and thresholds. 
stress state for tension/compression loading $\left(\sigma_{1}=F_{1 t, c}\right)$ and substituting Equation (30) in (40), with $\widetilde{C}=[\widetilde{E}]^{-1}$ the following equations hold

$$
\begin{aligned}
& \left(J_{11}^{H}\right)^{1 / 2} \frac{\widetilde{C}_{11}}{\left(1-d_{1 j}\right)^{2}\left(1+h_{1 j}\right)^{3}} F_{1 i}^{2}+\left(\left|H_{11}^{H} \cdot \frac{\widetilde{C}_{11}}{\left(1-d_{1 j}\right)^{2}\left(1+h_{1 j}\right)^{3}} F_{1 j}^{2}\right|\right)^{1 / 2} \\
& =1, \quad j=t, c
\end{aligned}
$$

in which $\left(\phi_{0}+\phi=1\right)$ is assumed to match the Tsai-Wu criterion at failure, $t$ and $c$ stand for tension and compression, respectively, $\left(d_{1 j}, h_{1 j}\right)$ represent the damage and healing parameters at failure. Equation (52) represents a nonlinear system from which $J_{11}^{H}$ and $H_{11}^{H}$ are determined in terms of experimental data $\left(F_{1}, d_{1 j}, h_{1 j}\right)$ with $j=t, c$.

Analogously, for transverse tension perpendicular to the fiber orientation, the following equation holds at failure load $\left(\sigma_{2}=F_{2 t}\right)$

$$
\left(J_{22}^{H}\right)^{1 / 2} \frac{\widetilde{C}_{22}}{\left(1-d_{2 t}\right)^{2}\left(1+h_{2 t}\right)^{3}} F_{2 t}^{2}+\left(\left|H_{2} \cdot \frac{\widetilde{C}_{22}}{\left(1-d_{2 t}\right)^{2}\left(1+h_{2 t}\right)^{3}} F_{2 t}^{2}\right|\right)^{1 / 2}=1
$$

which provide a relationship between the transverse components $J_{22}^{H}$ and $H_{22}^{H}$ of the characteristic healing tensor. Along the in-plane and outof-plane shear directions $\left(\sigma_{4}=F_{4}, \sigma_{5}=F_{5}, \sigma_{6}=F_{6}\right)$, the healing surface projected at failure into the stress space leads to the following equations

$$
\begin{aligned}
& \left(\frac{J_{11}^{H}}{\left(\Omega_{1 s}^{H}\right)^{2}}+\frac{J_{22}^{H}}{\left(\Omega_{2 s}^{H}\right)^{2}}\right)^{1 / 2} \frac{\widetilde{C}_{66} F_{6}^{2}}{\Omega_{1 s}^{D} \Omega_{2 s}^{D} \Omega_{1 s}^{H} \Omega_{2 s}^{H}}+\left(\left|\frac{H_{1}^{H}}{\Omega_{1 s}^{H}}+\frac{H_{2}^{H}}{\Omega_{2 s}^{H}}\right| \frac{\widetilde{C}_{66} F_{6}^{2}}{\Omega_{1 s}^{D} \Omega_{2 s}^{D} \Omega_{1 s}^{H} \Omega_{2 s}^{H}}\right)^{1 / 2}=1 \\
& \left(\frac{J_{11}^{H}}{\left(\Omega_{1 s}^{H}\right)^{2}}+\frac{J_{33}^{H}}{\left(\Omega_{3 s}^{H}\right)^{2}}\right)^{1 / 2} \frac{\widetilde{C}_{55} F_{5}^{2}}{\Omega_{1 s}^{D} \Omega_{3 s}^{D} \Omega_{1 s}^{H} \Omega_{3 s}^{H}}+\left(\left|\frac{H_{1}^{H}}{\Omega_{1 s}^{H}}+\frac{H_{3}^{H}}{\Omega_{3 s}^{H}}\right| \frac{\widetilde{C}_{55} F_{5}^{2}}{\Omega_{1 s}^{D} \Omega_{3 s}^{D} \Omega_{1 s}^{H} \Omega_{3 s}^{H}}\right)^{1 / 2}=1 \\
& \left(\frac{J_{22}^{H}}{\left(\Omega_{2 s}^{H}\right)^{2}}+\frac{J_{33}^{H}}{\left(\Omega_{3 s}^{H}\right)^{2}}\right)^{1 / 2} \frac{\widetilde{C}_{44} F_{4}^{2}}{\Omega_{2 s}^{D} \Omega_{3 s}^{D} \Omega_{2 s}^{H} \Omega_{3 s}^{H}}+\left(\left|\frac{H_{2}^{H}}{\Omega_{2 s}^{H}}+\frac{H_{3}^{H}}{\Omega_{3 s}^{H}}\right| \frac{\widetilde{C}_{44} F_{4}^{2}}{\Omega_{2 s}^{D} \Omega_{3 s}^{D} \Omega_{2 s}^{H} \Omega_{3 s}^{H}}\right)^{1 / 2}=1
\end{aligned}
$$

in which the components of the integrity tensor are $\Omega_{j s}^{H}=\left(1+h_{j s}\right)$ and $\Omega_{i s}^{D}=\left(1-d_{j s}\right)$ with $j=1,3$. Here $d_{j s}, h_{j s}$, represent the damage and healing at shear failure. Since shear strength are independent of the shear stress, Equations (54) have to be sign independent. Therefore, the linear terms have 
to be zero

$$
\left|\frac{H_{1}^{H}}{\Omega_{1 s}^{H}}+\frac{H_{2}^{H}}{\Omega_{2 s}^{H}}\right|=0, \quad\left|\frac{H_{1}^{H}}{\Omega_{1 s}^{H}}+\frac{H_{3}^{H}}{\Omega_{3 s}^{H}}\right|=0, \quad\left|\frac{H_{2}^{H}}{\Omega_{2 s}^{H}}+\frac{H_{3}^{H}}{\Omega_{3 s}^{H}}\right|=0,
$$

which introduce relationships between the in-plane and out-of-plane $\boldsymbol{H}$ components. Moreover, introducing scalar parameters $r_{s H}^{j}$ with $(j=12$, 13,23), Equation (55) can be written as

$$
H_{2}=-r_{s H}^{12} H_{1}, \quad H_{3}=-r_{s H}^{13} H_{1}, \quad H_{2}=-r_{s H}^{23} H_{3}
$$

with

$$
r_{s H}^{12}=\frac{\left(1+h_{2 s}\right)}{\left(1+h_{1 s}\right)} ; \quad r_{s H}^{13}=\frac{\left(1+h_{3 s}\right)}{\left(1+h_{1 s}\right)} ; \quad r_{s H}^{23}=\frac{\left(1+h_{3 s}\right)}{\left(1+h_{2 s}\right)}
$$

The $r_{s H}^{j}$ terms represent the scalar ratio between principal healing eigenvalues. Physically, they represent the availability of healing agent and mathematically, the ultimate shape of the healing domain. The stressstrain relationships at failure in the effective reference frame $C_{D H}$ is described by Equation (28). In particular, the shear components can be written as

$$
\frac{\tilde{\sigma}_{6}}{\widetilde{\gamma}_{6 \mathrm{ult}}}=\frac{G_{12}^{\mathrm{ult}}}{k_{s D}^{12} k_{s H}^{12}}, \quad \frac{\widetilde{\sigma}_{5}}{\widetilde{\gamma}_{5 \mathrm{ult}}}=\frac{G_{13}^{\mathrm{ult}}}{k_{s D}^{13} k_{s H}^{13}}, \quad \frac{\widetilde{\sigma}_{4}}{\widetilde{\gamma}_{4 \mathrm{ult}}}=\frac{G_{23}^{\mathrm{ult}}}{k_{s D}^{23} k_{s H}^{23}}
$$

with

$$
\begin{array}{ll}
k_{s D}^{12}=\left(1-d_{1 s}\right)\left(1-d_{2 s}\right), & k_{s H}^{12}=\left(1+h_{1 s}\right)\left(1+h_{2 s}\right) \\
k_{s D}^{13}=\left(1-d_{1 s}\right)\left(1-d_{3 s}\right), & k_{s H}^{13}=\left(1+h_{1 s}\right)\left(1+h_{3 s}\right) \\
k_{s D}^{23}=\left(1-d_{2 s}\right)\left(1-d_{3 s}\right), & k_{s H}^{23}=\left(1+h_{2 s}\right)\left(1+h_{3 s}\right)
\end{array}
$$

For example, $k_{s H}^{i j}$ with $(j=12,13,23)$ represent the ratio between damaged-healed stiffness at failure and damaged (not healed) stiffness at failure $G_{i z}^{\text {Healed }} / G_{R}^{\text {Damaged }}$. These parameters are related to the density of microcapsules and catalyst. Numerical evaluation is obtained by simple shear tests, which yield the product of healing and damage values at failure, simply as the ratio between ultimate to virgin shear modulus (Equations (58)). Introducing Equations (59) in Equations (54) the 
following equations hold

$$
\begin{aligned}
& \sqrt{\frac{J_{11}^{H} r_{s H}^{12}}{k_{s H}^{12}}+\frac{J_{22}^{H}\left[r_{s H}^{12}\right]}{k_{s H}^{12} r_{s H}^{12}}} \frac{\widetilde{C}_{66}}{k_{s H}^{12} k_{s D}^{12}} F_{6}^{2}=1 \\
& \sqrt{\frac{J_{11}^{H} r_{s H}^{13}}{k_{s H}^{13}}+\frac{J_{33}^{H}\left[r_{s H}^{13}\right]}{k_{s H}^{13} r_{s H}^{13}}} \frac{\widetilde{C}_{55}}{k_{s H}^{13} k_{s D}^{13}} F_{5}^{2}=1 \\
& \sqrt{\frac{J_{22}^{H} r_{s H}^{23}}{k_{s H}^{23}}+\frac{J_{33}^{H}\left[r_{s H}^{23}\right]}{k_{s H}^{23} r_{s H}^{23}}} \frac{\widetilde{C}_{44}}{k_{s H}^{23} k_{s D}^{23}} F_{4}^{2}=1
\end{aligned}
$$

Finally, Equations (53), (60), and Equation (56) describe a nonlinear system of equations in the unknown quantities $r_{s}^{j}(j=12,13,23)$ and $\left(H_{2}^{H}, H_{3}^{H}\right.$, $J_{22}^{H}, J_{33}^{H}$ ), from which healing characteristic tensors are completely identified in terms of experimental data.

\section{RESULTS AND DISCUSSION}

The identification procedure for damage and plasticity at the mesoscale level requires experimental data based on the single composite lamina behavior (Barbero and Lonetti, 2001, 2002; Lonetti et al., 2003). The strength values for different directions (longitudinal, transverse, in-plane/ out-of-plane shear) and the critical damage values yield a nonlinear system in which the unknown parameters are the damage characteristic tensor components, $J_{i j}^{D}$ and $H_{i}^{D}$. Basically, the same procedure is proposed here to identify the healing domain. The central assumption is that the $g^{h}$-function has the same analytical expression of the damage one. Therefore, the surface shape is mainly controlled by healing critical values and the characteristic tensors $\boldsymbol{J}^{H}$ and $\boldsymbol{H}^{H}$.

From the experimental point of view, damage develops into microcracks. Subsequently, these cracks reach the microcapsules and the healing agent is released. Numerically, the process is described by a healing domain, which controls the onset of healing. The evolution of such domain is defined by the normality rule, triggered when the driving thermodynamic healing forces reach the $g^{h}$-surface.

Healing and damage are shown schematically in Figure 4, in which $g^{d}$ with $\gamma+\gamma_{0}=1$ and $g^{h}$ with $\phi=0$ represent the damage and healing surfaces at failure and at the beginning of the process, respectively. The healing threshold $\phi_{0}$ defines when the process starts and it is dependent on the density of microcapsules and catalyst. Moreover, the healing critical 


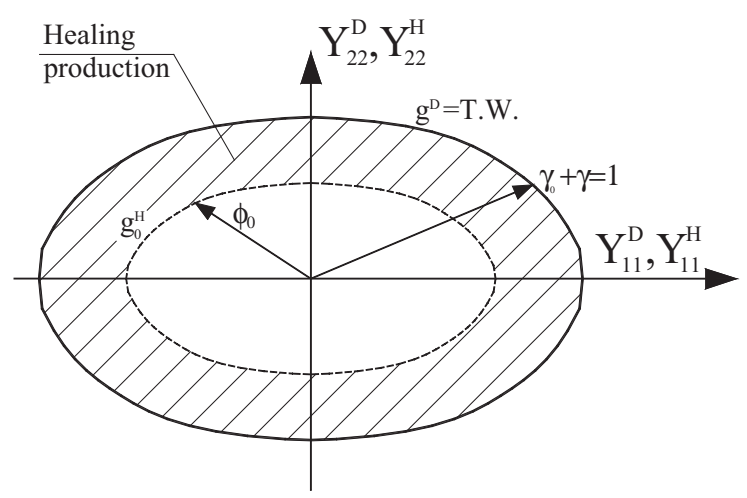

Figure 4. Healing and damage surfaces.

Table 2. Healing parameters.

\begin{tabular}{lc}
\hline Parameters & T300-5208 \\
\hline$J_{11}^{H}, J_{22}^{H}$ & $0.3885 \mathrm{e}-15,0.3522 \mathrm{e}-11$ \\
$H_{1}^{H}, H_{2}^{H}$ & $0.4255 \mathrm{e}-7,-0.1046 \mathrm{e}-6$ \\
$c_{1}^{h}, c_{2}^{h}, \phi$ & $0.15,-0.1 \mathrm{e}+05,0.4$ \\
$h_{1 c}, h_{2 c}=h_{3 c}$ & $0.1,0.5$ \\
\hline
\end{tabular}

eigenvalues $h_{1 c}, h_{2 c}, h_{3 c}$, represent the maximum allowable values of healing, which are to be obtained by experimental procedures. From the experimental point of view, microcapsule and catalyst density control two different phenomena: the beginning and the efficiency of the healing process. The critical healing values and the healing threshold controls both phenomena. From the numerical point of view, the latter control the beginning of the process, whereas the former defines the maximum size of the healing surface and the allowable values of the healing tensor. Only numerical results in terms of sensitivity analysis are shown in this work, but an experimental investigation has been initiated (Barbero et al., 2004) to properly define both values.

Lacking experimental data for the healing process, the model has been used to demonstrate the effect of healing on Carbon-Epoxy T300-5208 (Herakovich, 1998) for which the damage behavior is well documented. The material parameters determined by the identification procedure described in "Identification of Material Parameters" are shown in Table 2. In Figures 5-7, the solid line represents the actual behavior without healing and the dotted line the predicted behavior with the healing turned off by setting a high value of healing threshold $\phi_{0}$. The material properties are shown in Table 1. The damage evolution parameters $c_{1}^{D}, c_{2}^{D}, \gamma_{0}$, and damage 


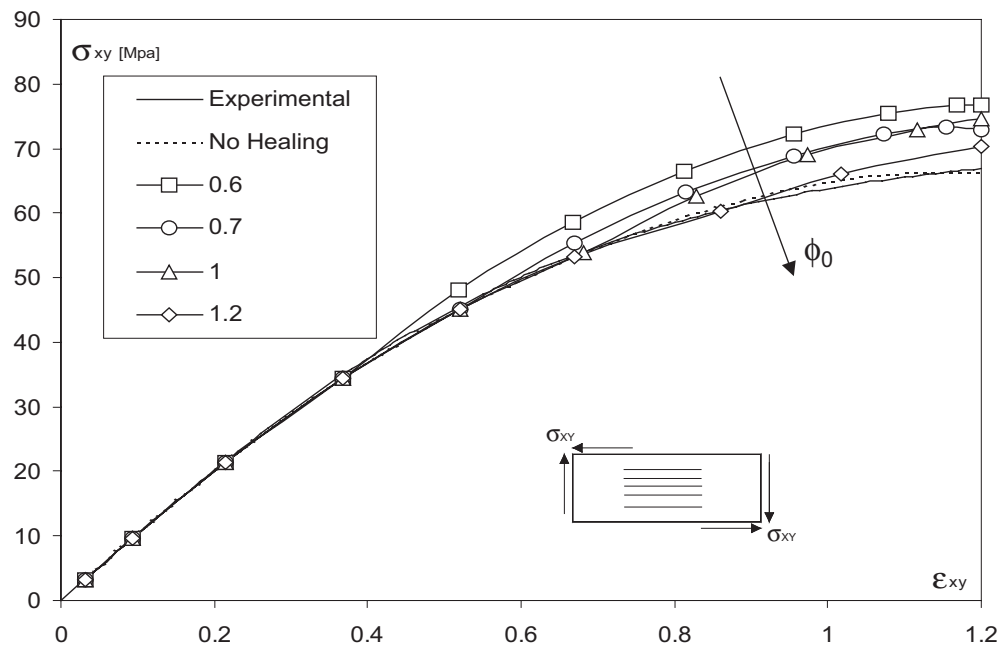

Figure 5. T300-5208 sensitivity of healing vs. healing threshold $\phi_{0}$ for an in-plane shear monotonic loading test with damage.

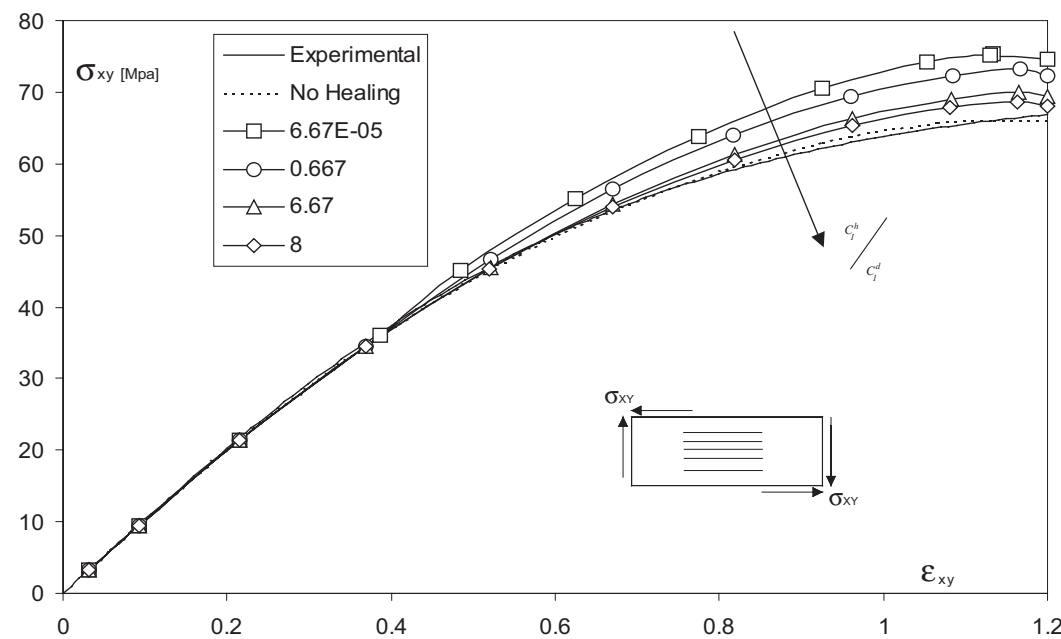

Figure 6. T300-5208 sensitivity of healing to healing evolution parameter $c_{1}^{h} / c_{1}^{d}$ for an in-plane shear monotonic loading test with damage.

characteristic tensors $\boldsymbol{J}^{D}, \boldsymbol{H}^{D}$ are identified in terms of available data for a single lamina as explained in Barbero and Lonetti (2001), using the material properties reported in the same reference. Subsequently, the healing phenomenon is evaluated assuming $\left(c_{1}^{h}=0.15, c_{2}^{h}=-0.1 E 5, \phi_{o}=0.4\right)$ as reference values. 


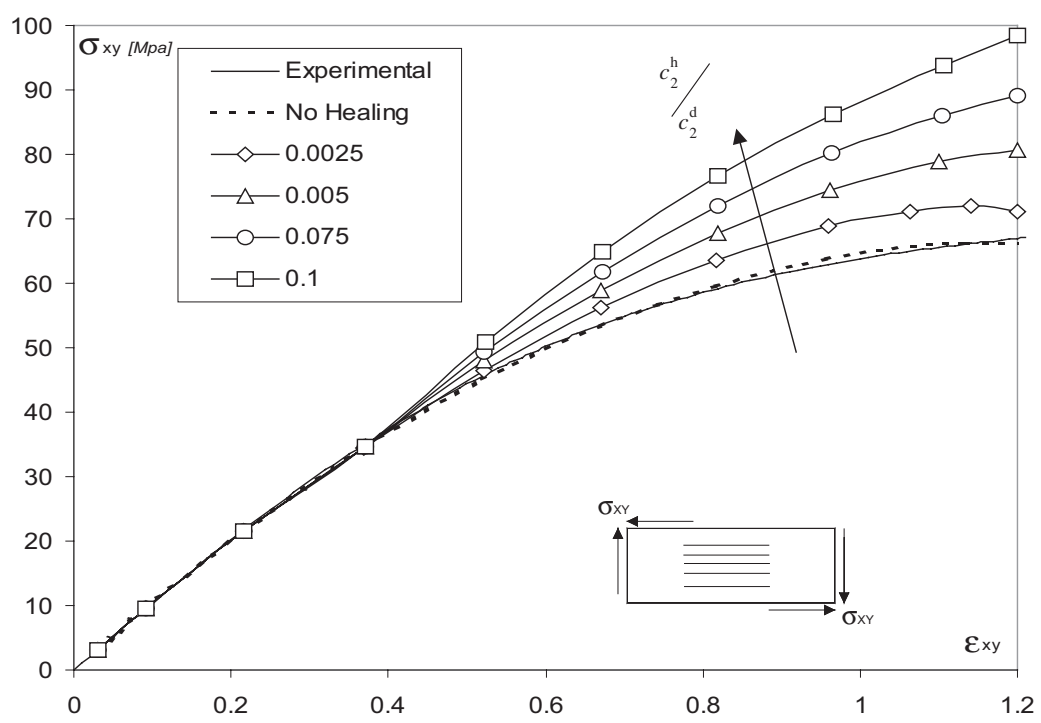

Figure 7. $T 300-5208$ sensitivity of healing to healing evolution parameter $c_{2}^{h} / c_{2}^{d}$ for an in-plane shear monotonic loading test with damage.

The in-plane shear stress-strain curve, as shown in Figures 5-7, highlights the stiffness improvement due to the healing effects. The sensitivity to healing of T300-5208 versus the healing threshold $\phi_{0}$ is shown in Figure 5 for an in-plane shear test under monotonic loading. The curve labeled "no healing" represents the prediction using actual material property data. It predicts accurately the experimental data because the T300-5208 material had no healing agent in it. The addition of healing clearly increases the shear stiffness and strength of the material. The sensitivity of T300-5208 versus evolution parameter $c_{1}^{h}$ is shown in Figure 6. The sensitivity of T300-5208 versus evolution parameter $c_{2}^{h}$ is shown in Figure 7. The parameters $c_{1}^{h}$ and $c_{2}^{h}$ control the evolution (hardening) of the healing domain. Increasing $c_{1}^{h}$ makes it harder to heal the material because the domain grows rapidly. This translates into less healing in Figure 6. The absolute value of $c_{2}^{h}$ controls the exponential decay in Equation (10) with larger absolute values resulting in more healing, as shown in Figure 7.

\section{CONCLUSIONS}

Continuum Damage Mechanics has been extended for the first time to incorporate healing process into what is called Continuum Damage Healing Mechanics (CDHM). Furthermore, the theory has been used to develop a specific model for fiber-reinforced polymer-matrix composites experiencing 
damage, plasticity, and healing. Expressions are given for the various domains, potentials, and evolution equations based on insight gained from experimental observations. A procedure for identifying the healing parameters is outlined. The procedure for integration of the evolution equations is given. Finally, the applicability of the theory and particular composite model is demonstrated by performing a parametric study of the effect of healing evolution parameters on the shear response of a material for which the nonhealing response is well known.

\section{APPENDIX I}

For a generic $(i+1)$ load step and $k$ iteration, the damage, plasticity, and healing functions at the first term of the Taylor expansion are written as

$$
\begin{aligned}
& g^{d} \cong g_{i+1}^{d}+\left.\frac{\partial g^{d}}{\partial \boldsymbol{Y}^{D}}\right|_{i+1} ^{k}\left(\boldsymbol{Y}_{i+1}^{D_{k+1}}-\boldsymbol{Y}_{i+1}^{D_{k}}\right)+\left.\frac{\partial g^{d}}{\partial \gamma}\right|_{i+1} ^{k}\left(\gamma_{i+1}^{k+1}-\gamma_{i+1}^{k}\right) \\
& g^{p} \cong g_{i+1}^{p}+\left.\frac{\partial g^{p}}{\partial \widetilde{\boldsymbol{\sigma}}}\right|_{i+1} ^{k}\left(\widetilde{\boldsymbol{\sigma}}_{i+1}^{k+1}-\widetilde{\boldsymbol{\sigma}}_{i+1}^{k}\right)+\left.\frac{\partial g^{p}}{\partial R}\right|_{i+1} ^{k}\left(R_{i+1}^{k+1}-R_{i+1}^{k}\right) \\
& g^{h} \cong g_{i+1}^{h}+\left.\frac{\partial g^{h}}{\partial \boldsymbol{Y}^{H}}\right|_{i+1} ^{k}\left(\boldsymbol{Y}_{i+1}^{H_{k+1}}-\boldsymbol{Y}_{i+1}^{H_{k}}\right)+\left.\frac{\partial g^{h}}{\partial \phi}\right|_{i+1} ^{k}\left(\phi_{i+1}^{k+1}-\phi_{i+1}^{k}\right)
\end{aligned}
$$

Using Equations (27)-(33), the thermodynamic forces $\left(\boldsymbol{Y}^{D}, \gamma, \tilde{\boldsymbol{\sigma}}, R, \boldsymbol{Y}^{H}, \phi\right)$ can be expressed as a function of internal kinematic variables $\left(\boldsymbol{D}, \delta, \boldsymbol{\varepsilon}^{p}, p\right.$, $\boldsymbol{H}, \mu)$ as

$$
\begin{aligned}
\Delta \boldsymbol{Y}^{D} \simeq & \left(\boldsymbol{Y}_{i+1}^{D_{k+1}}-\boldsymbol{Y}_{i+1}^{D_{k}}\right)=\left.\frac{\partial Y^{D}}{\partial \boldsymbol{D}}\right|_{i+1} ^{k}\left(\boldsymbol{D}_{i+1}^{k+1}-\boldsymbol{D}_{i+1}^{k}\right) \\
+ & \left.\frac{\partial Y^{D}}{\partial \boldsymbol{\varepsilon}^{p}}\right|_{i+1} ^{k}\left(\boldsymbol{\varepsilon}_{i+1}^{p(k+1)}-\boldsymbol{\varepsilon}_{i+1}^{p(k)}\right)+\left.\frac{\partial Y^{H}}{\partial \boldsymbol{H}}\right|_{i+1} ^{k}\left(\boldsymbol{H}_{i+1}^{k+1}-\boldsymbol{H}_{i+1}^{k}\right) \\
& \Delta \gamma \simeq\left(\gamma_{i+1}^{k+1}-\gamma_{i+1}^{k}\right)=\left.\frac{\partial \gamma}{\partial \delta}\right|_{i+1} ^{k}\left(\delta_{i+1}^{k+1}-\delta_{i+1}^{k}\right) \\
\left(\widetilde{\boldsymbol{\sigma}}_{i+1}^{(k+1)}-\widetilde{\boldsymbol{\sigma}}_{i+1}^{(k)}\right)= & \boldsymbol{M}^{-1}\left(\boldsymbol{D}_{i+1}^{k}\right)\left[\left.\frac{\partial \boldsymbol{\sigma}}{\partial \boldsymbol{\varepsilon}^{p}}\right|_{i+1} ^{k}\left(\boldsymbol{\varepsilon}_{i+1}^{p(k+1)}-\boldsymbol{\varepsilon}_{i+1}^{p(k)}\right)+\left.\frac{\partial \boldsymbol{\sigma}}{\partial \boldsymbol{D}}\right|_{i+1} ^{k}\left(\boldsymbol{D}_{i+1}^{k+1}-\boldsymbol{D}_{i+1}^{k}\right)\right. \\
& \left.+\left.\frac{\partial \boldsymbol{\sigma}}{\partial \boldsymbol{H}}\right|_{i+1} ^{k}\left(\boldsymbol{H}_{i+1}^{k+1}-\boldsymbol{H}_{i+1}^{k}\right)\right]
\end{aligned}
$$




$$
\begin{gathered}
\left(R_{i+1}^{k+1}-R_{i+1}^{k}\right)=\left.\frac{\partial R}{\partial p}\right|_{i+1} ^{k}\left(p_{i+1}^{k+1}-p_{i+1}^{k}\right) \\
\Delta \boldsymbol{Y}^{H} \simeq\left(\boldsymbol{Y}_{i+1}^{H_{k+1}}-\boldsymbol{Y}_{i+1}^{H_{k}}\right)=\left.\left.\frac{\partial \boldsymbol{Y}^{H}}{\partial \boldsymbol{H}}\right|_{i+1} ^{k}\left(\boldsymbol{H}_{i+1}^{k+1}-\boldsymbol{H}_{i+1}^{k}\right) \frac{\partial \boldsymbol{Y}^{H}}{\partial \boldsymbol{\varepsilon}^{p}}\right|_{i+1} ^{k}\left(\boldsymbol{\varepsilon}_{i+1}^{p(k+1)}-\boldsymbol{\varepsilon}_{i+1}^{p(k)}\right) \\
+\left.\frac{\partial \boldsymbol{Y}^{H}}{\partial \boldsymbol{D}}\right|_{i+1} ^{k}\left(\boldsymbol{D}_{i+1}^{k+1}-\boldsymbol{D}_{i+1}^{k}\right) \\
\Delta \phi \simeq\left(\phi_{i+1}^{k+1}-\phi_{i+1}^{k}\right)=\left.\frac{\partial \phi}{\partial \mu}\right|_{i+1} ^{k}\left(\mu_{i+1}^{k+1}-\mu_{i+1}^{k}\right)
\end{gathered}
$$

Moreover, the incremental expressions of the kinematic variables using Equations (38) are expressed as

$$
\begin{gathered}
\left(\boldsymbol{D}_{i+1}^{k+1}-\boldsymbol{D}_{i+1}^{k}\right)=\left.\Delta \lambda^{d} \frac{\partial f^{d}}{\partial \boldsymbol{Y}^{D}}\right|_{i+1} ^{k}, \quad\left(\delta_{i+1}^{k+1}-\delta_{i+1}^{k}\right)=-\Delta \lambda^{d} \\
\left(\widetilde{\boldsymbol{\varepsilon}}_{i+1}^{p(k+1)}-\widetilde{\boldsymbol{\varepsilon}}_{i+1}^{p(k)}\right)=\left.\Delta \lambda^{p} \frac{\partial g^{p}}{\partial \widetilde{\boldsymbol{\sigma}}}\right|_{i+1} ^{k}, \quad\left(p_{i+1}^{(k+1)}-p_{i+1}^{(k)}\right)=-\Delta \lambda^{p} \\
\left(\boldsymbol{H}_{i+1}^{k+1}-\boldsymbol{H}_{i+1}^{k}\right)=\left.\Delta \lambda^{h} \frac{\partial f^{h}}{\partial \boldsymbol{Y}^{H}}\right|_{i+1} ^{k}, \quad\left(\mu_{i+1}^{k+1}-\mu_{i+1}^{k}\right)=-\Delta \lambda^{h}
\end{gathered}
$$

Finally, introducing Equations (A8)-(A10) and (A2)-(A7) into Equations (A1), a system of equations is obtained, in which $\Delta \lambda^{i}$ are the unknown quantities

$$
\begin{aligned}
& g^{d}\left(\lambda^{p}, \lambda^{d}, \lambda^{h}\right) \cong g_{i+1}^{d}+\left.\frac{\partial g^{d}}{\partial \lambda^{d}}\right|_{i+1} ^{k} \Delta \lambda^{d}+\left.\frac{\partial g^{d}}{\partial \lambda^{p}}\right|_{i+1} ^{k} \Delta \lambda^{p}+\left.\frac{\partial g^{d}}{\partial \lambda^{h}}\right|_{i+1} ^{k} \Delta \lambda^{h} \\
& g^{p}\left(\lambda^{p}, \lambda^{d}, \lambda^{h}\right) \cong g_{i+1}^{p}+\left.\frac{\partial g^{p}}{\partial \lambda^{d}}\right|_{i+1} ^{k} \Delta \lambda^{d}+\left.\frac{\partial g^{p}}{\partial \lambda^{p}}\right|_{i+1} ^{k} \Delta \lambda^{p}+\left.\frac{\partial g^{p}}{\partial \lambda^{h}}\right|_{i+1} ^{k} \Delta \lambda^{h} \\
& g^{h}\left(\lambda^{p}, \lambda^{d}, \lambda^{h}\right) \cong g_{i+1}^{h}+\left.\frac{\partial g^{h}}{\partial \lambda^{d}}\right|_{i+1} ^{k} \Delta \lambda^{d}+\left.\frac{\partial g^{h}}{\partial \lambda^{p}}\right|_{i+1} ^{k} \Delta \lambda^{p}+\left.\frac{\partial g^{h}}{\partial \lambda^{h}}\right|_{i+1} ^{k} \Delta \lambda^{h}
\end{aligned}
$$

where

$$
\frac{\partial g^{d}}{\partial \lambda^{d}}=\left(\frac{\partial g^{d}}{\partial \boldsymbol{Y}^{D}} \frac{\partial \boldsymbol{Y}^{D}}{\partial \boldsymbol{D}} \frac{\partial f^{d}}{\partial \boldsymbol{Y}^{D}}-\frac{\partial g^{d}}{\partial \gamma} \frac{\partial \gamma}{\partial \delta}\right)
$$




$$
\begin{gathered}
\frac{\partial g^{d}}{\partial \lambda^{p}}=\left(\frac{\partial g^{d}}{\partial \boldsymbol{Y}^{D}} \frac{\partial \boldsymbol{Y}^{D}}{\partial \boldsymbol{\varepsilon}^{p}} \boldsymbol{M}^{-1} \frac{\partial g^{p}}{\partial \widetilde{\boldsymbol{\sigma}}}\right) \\
\frac{\partial g^{d}}{\partial \lambda^{h}}=\left(\frac{\partial g^{h}}{\partial \boldsymbol{Y}^{D}} \frac{\partial \boldsymbol{Y}^{D}}{\partial \boldsymbol{H}} \frac{\partial f^{f}}{\partial \boldsymbol{Y}^{H}}\right) \\
\frac{\partial g^{p}}{\partial \lambda^{d}}=\frac{\partial g^{p}}{\partial \widetilde{\boldsymbol{\sigma}}} \frac{\partial}{\partial \boldsymbol{D}}\left[\boldsymbol{M}^{-1} \boldsymbol{\sigma}\right] \frac{\partial f^{d}}{\partial \boldsymbol{Y}^{D}} \\
\frac{\partial g^{p}}{\partial \lambda^{p}}=\left(\frac{\partial g^{p}}{\partial \widetilde{\boldsymbol{\sigma}}} \boldsymbol{M}^{-1} \frac{\partial \sigma}{\partial \boldsymbol{\varepsilon}^{p}} \boldsymbol{M}^{-1} \frac{\partial g^{p}}{\partial \widetilde{\boldsymbol{\sigma}}}-\frac{\partial g^{p}}{\partial R} \frac{\partial R}{\partial p}\right) \\
\frac{\partial g^{p}}{\partial \lambda^{h}}=\frac{\partial g^{p}}{\partial \widetilde{\boldsymbol{\sigma}}} \frac{\partial}{\partial \boldsymbol{H}}\left[\boldsymbol{M}^{-1} \boldsymbol{\sigma}\right] \frac{\partial f^{h}}{\partial \boldsymbol{Y}^{h}} \\
\frac{\partial g^{h}}{\partial \lambda^{h}}=\left(\frac{\partial g^{h}}{\partial \boldsymbol{Y}^{H}} \frac{\partial \boldsymbol{Y}^{H}}{\partial \boldsymbol{H}} \frac{\partial f^{h}}{\partial \boldsymbol{Y}^{h}}-\frac{\partial g^{h}}{\partial \phi} \frac{\partial \phi}{\partial \mu}\right), \\
\frac{\partial g^{h}}{\partial \lambda^{p}}=\left(\frac{\partial g^{h}}{\partial \boldsymbol{Y}^{H}} \frac{\partial \boldsymbol{Y}^{H}}{\partial \boldsymbol{\varepsilon}^{p}} \boldsymbol{M}^{-1} \frac{\partial g^{p}}{\partial \widetilde{\boldsymbol{\sigma}}}\right), \\
\frac{\partial g^{h}}{\partial \lambda^{d}}=\left(\frac{\partial g^{h}}{\partial \boldsymbol{Y}^{H}} \frac{\partial \boldsymbol{Y}^{H}}{\partial \boldsymbol{D}} \frac{\partial f^{d}}{\partial \boldsymbol{Y}^{d}}\right),
\end{gathered}
$$

\section{REFERENCES}

Aboudi, J. (1991). Mechanics of Composite Materials: A Unified Approach, Elsevier, New York.

Abu Al-Rub, R.K. and Voyiadjis, G.Z. (2003). On the Coupling of Anisotropic Damage and Plasticity Models for Ductile Materials, International Journal of Solids and Structures, 40(11): 2611-2643.

Adam, J.L. (1999). A Simplified Model of Wound Healing (with Particular Reference to the Critical Size Defect), Mathematical and Computer Modelling, 30(5-6), 23-32.

Ando, Kotoji, Chu, Min-Cheol, Tsuji, Kiichi, Hirasawa, Toshikazu, Kobayashi, Yasuyoshi and Sato, Shigemi (2002a). Crack Healing Behaviour and High-temperature Strength of Mullite/SiC Composite Ceramics, Journal of the European Ceramic Society, 22(8): 1313-1319.

Ando, Kotoji, Chu, Min-Cheol, Tsuji, Kiichi, Hirasawa, Toshikazu, Kobayashi, Yasuyoshi and Sato, Shigemi (2002b). Crack-healing Behavior of $\mathrm{Si}_{3} \mathrm{~N}_{4} / \mathrm{SiC}$ Ceramics Under Stress and Fatigue Strength at the Temperature of Healing $\left(1000^{\circ} \mathrm{C}\right)$, Journal of the European Ceramic Society, 22(8): 1339-1346.

Barbero, E.J. (1999). Introduction to Composite Materials Design, Taylor and Francis, N.Y.

Barbero, E.J. and DeVivo, L. (2001). A Constitutive Model for Elastic Damage in Fiberreinforced PMC Laminae, J. of Damage Mechanics, 10(1): 73-93.

Barbero, E.J. and Lonetti, P. (2001). Damage Model for Composites Defined in Terms of Available Data, Mechanics of Composite Materials and Structures, 8(4): 299-315.

Barbero, E.J. and Lonetti, P. (2002). An Inelastic Damage Model for Fiber Reinforced Laminates, Journal of Composite Material, 36(8): 941-962.

Barbero, E.J. and Lonetti, P. (2003). Application of Continuum Damage Healing Mechanics To Self-healing Composites, ASME IMECE 2003-43738, Washington, DC, November. 
Barbero, E.J., Ford, K. and Stiller, A. (2004) Continuum Damage Healing Mechanics for Modeling of Self-healing Composites, Int. SAMPE Congress, CA, April.

Brown, E.N., Sottos, N.R. and White, S.R. (2002). Fracture Testing of Self-Healing Polymer Composite, Experimental Mechanics, 42(4): 372-379.

Chaboche, J.L. (1988). Continuum Damage Mechanics: Part I: General Concept, Part II Damage Growth, Crack Initiation and Crack Growth, J. Appl. Mech., 55(3): 59-71.

Chow, C.L. and Wang, J. (1987). An Anisotropic Theory of Elasticity for Continuum Damage, Mechanics, Int. J. Damage Mech., 4(3): 251-263.

Coleman, B.D. and Gurtin, M.E. (1967). Thermodynamics with Internal State Variables, J. Chem. Phys., 47: 597-613.

Cordebois, J.P. and Sidoroff, F. (1979). Damage Induced Elastic Anisotropy, Coll.Euromech 115, Villard De Lans, Also In: Boehler (ed.), (1983) Mechanical Behavior of Anisotropic Solids, Martinus Nijhoff, Boston, pp. 761-774.

Crisfield, M.A. (1991). Non-linear Finite Element Analysis of Solids and Structures, Vol. 1, John Wiley \& Sons, NY, USA.

Dvorak, G.J. (2000). Composite Materials: Inelastic Behavior, Damage, Fatigue and Fracture, International Journal of Solids and Structures, 37: 155-170.

Gong, Xl, Gong, Xj, Laksimi, A. and Benzeggagh, M.L. (2000). Application of Tsai-Wu Criterion to Notched and Unnotched Composite Laminates under Torque Loading, Journal of Composite Materials, 34(6): 460-478.

Hansen, N. and Schreyer, H.L. (1994). A Thermodynamic Consistent Framework for Theories of Elastoplasticity Coupled with Damage, Int. J. Solids Structures, 31: 359-389.

Herakovich, C.T. (1998). Mechanics of Fibrous Composites, John Wiley, N.Y.

Jacobsen, S. and Sellevold, E.J. (1996). Self Healing of High Strength Concrete after Deterioration by Freeze/Thaw, 26(1): January, 55-62.

Jacobsen, S., Marchand, J. and Boisvert, L. (1996). Effect of Cracking and Healing on Chloride Transport in OPC Concrete, Cement and Concrete Research, 26(6): 869-881.

Ju, J.U. (1989). On Energy Based Coupled Elastoplastic Damage Theories: Constitutive Modeling and Computational Aspects, Int. J. Solids Structures, 25: 803-833.

Kessler, M.R. and White, S.R. (2001). Self-Activated Healing of Delamination Damage in Woven Composites, Composites Part A: Applied Science and Manufacturing, 32(5): 683-699.

Ladeveze, P. and Le Dantec, E. (1992). Damage Modelling of the Elementary Ply for Laminated Composites, Composites Science and Technology, 43: 257-267.

Liu, Y.M., Michell, T.E. and Wadley, H.N.G. (1997). Anisotropic Damage Evolution in Unidirectional Fiber Reinforced Ceramics, Acta Materialia, 45(10): 3981-3992.

Lonetti, P., Barbero, E.J., Zinno, R. and Greco, F. (2003). Interlaminar Damage for Fiber Reinforced Polymeric Matrix Composite, Journal of Composite Materials, 37(16): $1485-1504$.

Lubliner, J. (1972). On the Thermodynamic Foundations of Nonlinear Solids Mechanics, Int. J. Non-Linear Mech., 7: 237-254.

Luccioni, B., Oller, S. and Danesi, R. (1996). Coupled Plastic-Damage Model, Computer Methods in Applied Mechanics and Engineering, 129: 81-89.

Miao, S., and Wang, M.L. (1994). Mechanical Properties of Consolidated Crushed Rock Salt, In: Proc., 4th Annu, WERC Technl. Development Conf., WERC, Dept. of Energy, Las Cruces, N.M.

Miao, S., Wang, M.L. and Schreyer, H.L. (1995). Constitutive Models for Healing of Materials with Application to Compaction of Crushed Rock Salt, Journal of Engineering Mechanics, 121(12): 1122-1129.

Murakami, S. (1988). Mechanical Modelling of Material Damage, J. Appl. Mech., 55(6): 280-286. 
Ogden (1983). Non Linear Elastic Deformation, Dover Pub., New York.

Piggott, M.R., Liu, K. and Wang, J. (2000). New Experiments Suggest That all the Shear and Some Tensile Failure Processes are Inappropriate Subjects for ASTM Standards, Astm Stp 1383, Composite Structures: Theory And Practice.

Pindera, M.J. (1992). Stress Analysis of Multilayered Anisotropic Elastic Media, J. Appl. Mech-T ASME, 59(1): 238-258.

Ramm, W. and Biscoping, M. (1998). Autogenous Healing and Reinforcement Corrosion of Water-Penetrated Separation Cracks in Reinforced Concrete, Nuclear Engineering and Design 179: 191-200.

Simpson, A.H.R.W., Gardner, T.N., Evans, M. and Kenwright, J. (2000). Stiffness, Strength and Healing Assessment in Different Bone Fractures — a Simple Mathematical Model, Injury, 31(10): December, 777-781.

Voyiadjis, G.Z. and Deliktas, B. (2000). A Coupled Anisotropic Damage Model for the Inelastic Response of Composite Materials, Comp. Methods in Appl. Mech. Eng., 183: 159-199.

White, S.R., Sottos, N.R., Geubelle, P.H., Moore, J.S., Kessler, M.R., Sriram, S.R. Brown, E.N. and Viswanathan, S. (2001). Autonomic Healing of Polymer Composites, Nature, 409: 794-797. 